\title{
The capabilities and limitations of conductance-based compartmental neuron models with reduced branched or unbranched morphologies and active dendrites
}

\author{
Eric B. Hendrickson • Jeremy R. Edgerton • \\ Dieter Jaeger
}

Received: 8 April 2010 /Revised: 24 May 2010 / Accepted: 22 June 2010 /Published online: 10 July 2010

(C) The Author(s) 2010. This article is published with open access at Springerlink.com

\begin{abstract}
Conductance-based neuron models are frequently employed to study the dynamics of biological neural networks. For speed and ease of use, these models are often reduced in morphological complexity. Simplified dendritic branching structures may process inputs differently than full branching structures, however, and could thereby fail to reproduce important aspects of biological neural processing. It is not yet well understood which processing capabilities require detailed branching structures. Therefore, we analyzed the processing capabilities of full or partially branched reduced models. These models were created by collapsing the dendritic tree of a full morphological model of a globus pallidus (GP) neuron while preserving its total surface area and electrotonic length, as well as its passive and active parameters. Dendritic trees were either collapsed into single cables (unbranched models) or the full complement of branch points was preserved (branched models). Both reduction strategies allowed us to compare dynamics between all models using the same channel density settings. Full model responses to somatic inputs were generally preserved by both types of reduced model while dendritic
\end{abstract}

\section{Action Editor: Frances K. Skinner \\ Electronic supplementary material The online version of this article (doi:10.1007/s10827-010-0258-z) contains supplementary material, which is available to authorized users.}

\section{E. B. Hendrickson}

Biomedical Engineering Department, Georgia Inst of Tech,

313 Ferst Dr,

Atlanta, GA 30332, USA

E. B. Hendrickson · J. R. Edgerton • D. Jaeger $(\square)$

Department of Biology, Emory University,

1510 Clifton Rd. NE,

Atlanta, GA 30322, USA

e-mail: djaeger@emory.edu input responses could be more closely preserved by branched than unbranched reduced models. However, features strongly influenced by local dendritic input resistance, such as active dendritic sodium spike generation and propagation, could not be accurately reproduced by any reduced model. Based on our analyses, we suggest that there are intrinsic differences in processing capabilities between unbranched and branched models. We also indicate suitable applications for different levels of reduction, including fast searches of full model parameter space.

Keywords Simulation · Computational modeling · Conductance $\cdot$ Morphology Globus pallidus .

Synaptic integration

\section{Introduction}

Single cell computer models can provide important insights into the mechanisms of neuronal processing. 'Full models' possessing morphologically complete dendritic trees have helped to elucidate many key functions of dendritic computation, for example by showing that the extent of action potential back propagation depends on the degree of dendritic branching (Schaefer et al. 2003). Furthermore, dendritic sub-branches have been shown to independently regulate channel densities (Losonczy et al. 2008). Due to high computational requirements, full models are frequently replaced in network simulations and even in the exploration of single neuron properties by 'reduced' models possessing fewer compartments and simplified dendritic branching structures (Herz et al. 2006). For example, reduced models were used to study the control of burst firing by dendritic NMDA receptor activation (Kuznetsov et al. 2006) and the 
reliance on dendritic T-currents for low-threshold $\mathrm{Ca}^{2+}$ spiking (Destexhe et al. 1998). Computationally efficient reduced models are also particularly useful for the simulation of large networks of neurons and have been used, for example, to study gamma-frequency synchronization through dendro-dendritic gap junctions (Traub et al. 2001).

While cable theory has shown that many features of properly constrained passive models can be analytically preserved by reduced structures (Burke 2000; Rall et al. 1992), some features of full passive models cannot be preserved by reduced morphologies. For instance, nonuniform synaptic distributions are by definition impossible to implement in reduced morphologies with a single dendritic compartment but have been shown to provide variation in both the resting membrane potential and the effectiveness of distal synaptic inputs (Holmes and Woody 1989). With the addition of voltage and calcium gated conductances in active models, the potential to lose processing capabilities when reducing morphological complexity only increases (Rall et al. 1992). Therefore, a clear understanding of the limitations of reduced active models with and without the preservation of the detailed branching structure of a full model is highly desirable in order to reach biologically valid conclusions regarding network computation.

In the present study, we used a previously characterized full GP neuron model (Gunay et al. 2008) to conduct a detailed analysis of the limitations of two types of model reduction strategies. The full GP model has extended thin dendrites with multiple active conductances, which makes it a good test case for possible limitations resulting from model reduction. We compared two principled strategies of model reduction that can be applied to full morphologies of all cell types. The first strategy preserved the detailed branching structure of the full model, while the second strategy only preserved the surface area and electronic length of each major dendritic branch by collapsing subbranches. We found that both branched and unbranched model morphologies could retain many properties such as spike waveforms and fI curves of the full model without adjusting channel densities. However, when the morphology was reduced to a small number of compartments, detailed spike waveforms showed pronounced mismatches due to differences in axial current flow. Model properties dependent on local dendritic interactions between inputs and active conductances could not be fully maintained even in models with branched dendrites due to unavoidable local input impedance mismatches between full and reduced models. Overall, our analysis provides clear guidelines to the limitations of reduced models and highlights specific aspects of synaptic processing requiring full branching structures. Furthermore, we show that our reduced models can successfully reproduce many integrative properties of full models.
Indeed, given the reduction strategies that we used, identical channel density settings can be used in both full and reduced morphologies to produce the similar dynamics. In particular, we show that our unbranched reduced models with $>10$ compartments can reproduce full model somatic input output dynamics, which allows them to be used as fast search engines of the full model parameter space.

\section{Methods}

\subsection{Full morphological GP neuron model}

Previously, we have described the construction and validation of a database of full GP models (Gunay et al. 2008). Models were constructed with three dendritic morphologies, which were obtained using Neurolucida (MicroBrightField, Inc., Williston, VT) reconstructions of biocytin filled rat GP neurons in slice. One of these morphologies (s34 in Gunay et al. 2008) was selected for the full GP model used in this study: this morphology contained a soma and 511 dendritic compartments. The passive parameters for this morphology were optimized in a previous study of ours in order to fit the responses of the recorded cell (s34 in Gunay et al. 2008) to brief somatic current injection pulses (Hanson et al. 2004). As in Hanson et al. 2004 and Gunay et al. 2008, the passive properties in all compartments were set to their optimized values for this morphology: $\mathrm{Rm}$ (specific membrane resistance) $=1.47 \Omega-\mathrm{m}^{2}, \mathrm{Cm}$ (specific membrane capacitance) $=0.024 \mathrm{~F} / \mathrm{m}^{2}$, and $\mathrm{Ra}$ (specific axial resistance) $=$ $1.74 \Omega-\mathrm{m}$. Each somatic and dendritic compartment of the full model also contained 11 different voltage and calcium gated ion channels: these were fast sodium $(\mathrm{NaF})$, persistent sodium $(\mathrm{NaP})$, slow activating delayed rectifier (Kv2), fast activating incompletely inactivating potassium $(\mathrm{Kv} 3)$, low voltage activated potassium $\left(\mathrm{Kv}_{\text {fast }}\right.$ and $\left.\mathrm{Kv} 4_{\text {slow }}\right), \mathrm{KCNQ}$ family potassium (KCNQ), calcium activated potassium (SK), high voltage activated calcium (CaHVA), and $\mathrm{H}$-current $\left(\mathrm{HCN}_{\text {fast }}\right.$ and $\mathrm{HCN}_{\text {slow }}$ ). All dendritic compartments were given the same conductance density for each channel (i.e. no gradients). A full description of channel kinetics is given in supplemental Tables S1 and S2 (Online Resource 1). See Gunay et al. 2008 for the process of parameter tuning.

Axons are generally not reconstructed from neurons recorded in brain slices, because the slicing procedure often severs the axon close to the soma. This was the case in our GP reconstructions as well, and therefore our study of full GP model properties employed a default axon comprised of multiple nodal and internodal segments (Gunay et al. 2008). Here, we replaced this artificial axon with a single axonal compartment so that it could be directly transferred to the reduced models without greatly increasing morphological 
complexity. This replacement required a recalibration of axonal and somatic channel densities (Supplemental Table S3, Online Resource 1) in order to preserve the original model activity that was tuned to match slice recordings.

The tuned parameter set used for most of this study contained a medium level of dendritic sodium conductance (gNa consisting of $40 \mathrm{~S} / \mathrm{m}^{2} \mathrm{NaF}$ and $1 \mathrm{~S} / \mathrm{m}^{2} \mathrm{NaP}$ ) which was not sufficient to support dendritic spike initiation. However, dendritic spike initiation has been recorded in GP (Hanson et al. 2004) and other neuron types, and represents an important possible mode of synaptic integration in vivo. Therefore, dendritic spike initiation and other responses were compared between the full and reduced models using an alternative to the tuned parameter set which contained a high level of dendritic $\mathrm{gNa}\left(800 \mathrm{~S} / \mathrm{m}^{2}\right.$ of dendritic $\mathrm{NaF}$ and $1 \mathrm{~S} / \mathrm{m}^{2}$ of dendritic NaP). This dendritic $\mathrm{gNa}$ density allowed frequent spike initiation with appropriate dendritic stimulation (Supplemental Fig. S3, Online Resource 1). To assess the contribution of both levels of dendritic $\mathrm{gNa}$ to mismatches between the full and reduced models, a second alternative parameter set was used which contained no dendritic gNa. Model responses with both alternative parameter sets were described in the text or shown in supplemental figures when there were important differences with the results using the tuned parameter set.

\subsection{Morphological reductions}

We collapsed the dendritic tree of the full GP model in order to study which full model activities could be matched by morphologically reduced models. Morphological reduction could lead to functional mismatches with the full model for two main reasons: first, by definition, morphological reduction must cause a decrease in the number of compartments and an increase in average compartment size that may not adequately represent voltage gradients in cylinders; second, morphological reduction could cause a loss of the detailed branching structure of the full model. To distinguish between these two possible causes of activity changes, two types of reduced models were created. The first type required dozens of compartments in order to preserve the detailed branching structure of the full model (Fig. 1(b), 'branched' reduced models), while the second type required only a few compartments because it did not preserve the detailed branching structure (Fig. 1(c), 'unbranched' reduced models).

To create the branched reduced models, groups of full model dendritic compartments which did not span branch points were identified: there were 39 such groups. Each group of compartments was converted into a single compartment such that the total surface area (TSA) and end to end electrotonic length (L) were preserved. TSA was

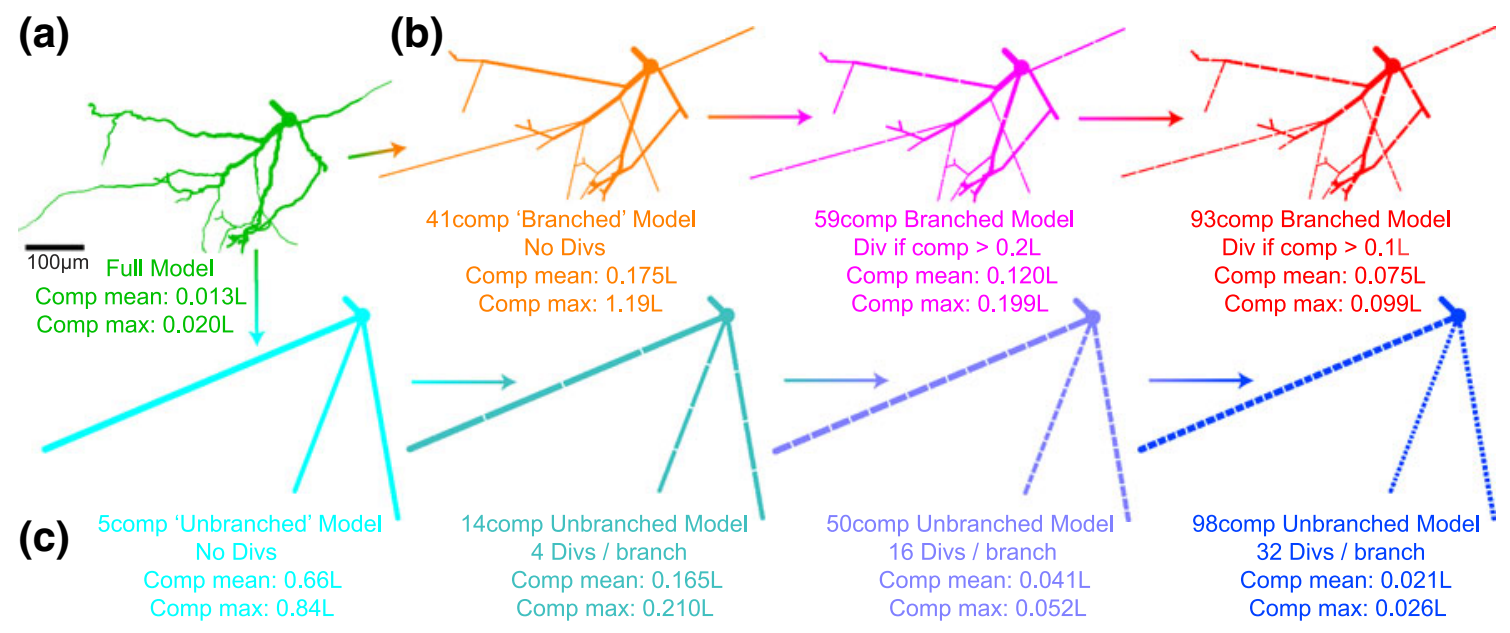

Fig. 1 Methods of morphological reduction. To aid visualization, dendritic diameters are multiplied by 5 while soma diameters are multiplied by 2. Mean and maximum compartment electrotonic lengths (L) are provided for each model to facilitate comparisons of structure across model types. (a) The full GP model (green) has 513 compartments: these include 511 dendritic compartments, an axon compartment, and a soma. (b) To create the simplest 'branched' reduced model (41comp, orange), the soma and axon compartment were unaltered while each group of compartments in the full model's dendritic tree not containing any branch points was collapsed into one dendritic compartment with the same total surface area and total electrotonic length (see Methods). The dendrites of the 41 comp model were then divided lengthwise into equal parts until no dendritic

compartment in the model was longer than $0.2 \mathrm{~L}$ (59comp, magenta) or $0.1 \mathrm{~L}$ (93comp, red). (c) To create the first 'unbranched' reduced model (5comp, cyan), each major branch of the full model's dendritic tree was collapsed into a single dendritic compartment which preserved the total surface area and average soma-to-tip electrotonic distance for that major branch (see Methods). To create the 14 comp (turquoise), 50comp (purple), and 98comp (blue) models, each dendritic compartment of the 5comp model was divided lengthwise into 4, 16, and 32 pieces, respectively. All conductance densities and passive parameters were preserved in each reduced model. The color scheme used in this figure was consistently applied to all remaining figures 
preserved in order to maintain active current densities and cell input resistance (Destexhe 2001; Tobin et al. 2006), while L was preserved because the electrotonic structure of a neuron strongly affects the integration of synaptic input and dendritic attenuation of somatic voltage signals (Burke 2000; Destexhe 2001). Holding all passive parameters including $\mathrm{Rm}$ and $\mathrm{Ra}$ fixed, the TSA and $\mathrm{L}$ for a given group were used to calculate the length (l) and radius (r) of the corresponding reduced model compartment using the following two equations:

$L=l \sqrt{\frac{2 * R a}{R m^{*} r}}$

$T S A=2 * \pi^{*} r^{*} l$

Including the soma, the axon compartment, and the 39 dendritic compartments generated this way, the simplest branched reduced model had 41 compartments (Fig. 1(b), '41comp' orange model). The 41comp model possessed some compartments that were quite electrotonically extended and could prevent the 41 comp model from matching certain full model responses. Therefore, the 41comp model's extended dendritic compartments were divided such that none was longer than $0.2 \mathrm{~L}$ (Fig. 1(b), '59comp' model) or 0.1 L (Fig. 1(b), '93comp' model).

While the branched reduced models maintained the TSA and $\mathrm{L}$ of all detailed branches of the full model, the unbranched reduced models only maintained the TSA and $\mathrm{L}$ of each of the three major branches. It is possible to maintain most passive electrical characteristics of a full model when reducing its branching structure if the dendritic tree meets two criteria: 1) dendrites must follow the $3 / 2$ power branching rule and 2) all paths from the soma to dendritic tips must have equal electrotonic lengths (Rall 1959, 1964). However, as for most real dendritic trees, these strict criteria were not satisfied by the GP full model. Therefore, we were forced to choose which aspects of the full model's morphology to preserve when reducing the morphology to produce the unbranched reduced models. We chose to preserve the TSA and average tip to tip L of each major branch for the same reasons that we preserved TSA and L for each branch in the branched reduced models.

For the unbranched reduced models, the TSA of each major branch was calculated by summing its compartment surface areas; the L of each major branch was estimated as the mean soma-to-tip L for all the tips in that major branch. Using Eqs. (1) and (2) and these values for TSA and L, we reduced each major branch of the full GP model into a single compartment to create a 5-compartment unbranched reduced model (Fig. 1(c), '5comp' model). Because each dendritic compartment of the 5 comp model was greater than $0.5 \mathrm{~L}$, we progressively divided each dendritic compartment lengthwise to be able to analyze which features of the full model were lost due to altered branching structure and which were due to increased compartment size: each compartment of the 5comp model was divided lengthwise into 4, 16 and 32 equal pieces to form the '14comp', '50comp' and '98comp' models, respectively (Fig. 1(c)).

\subsection{Synaptic inputs}

General features In several simulations, randomly timed synaptic events were applied to dendritic compartments. Excitatory synaptic inputs consisted of mixed AMPA $\left(\tau_{\text {rise }}=1 \mathrm{~ms}, \tau_{\text {fall }}=3 \mathrm{~ms}, E_{\mathrm{syn}}=0 \mathrm{mV}\right)$ and NMDA $\left(\tau_{\text {rise }}=\right.$ $10 \mathrm{~ms}, \tau_{\text {fall }}=30 \mathrm{~ms}, E_{\mathrm{syn}}=0 \mathrm{mV}$ ) conductances, while inhibitory inputs used solely GABA-A $\left(\tau_{\text {rise }}=1 \mathrm{~ms}, \tau_{\text {fall }}=\right.$ $12 \mathrm{~ms}, E_{\mathrm{syn}}=-80 \mathrm{mV}$ ) conductance. All synapses had a maximal conductance of $0.25 \mathrm{nS}$ and were distributed in the full model such that each dendritic compartment received one AMPA, one NMDA and one GABA-A synapse. In the branched reduced models, synapses were directly mapped from the full model and preserved both their locations in the detailed branching structure and their exact activation times. In the unbranched reduced models, synapses could only be approximately mapped: synapses were placed in compartments such that the electrotonic span and major branch location of each compartment determined how many synaptic inputs it received. For example, the most proximal dendritic compartment in the 14 comp model's (Fig. 1(c)) first branch spanned from 0 to $0.14 \mathrm{~L}$. In the full model's first major branch, this electrotonic span contained 20 compartments; therefore, the most proximal dendritic compartment in the 14comp model's first branch received the synapses contained in those 20 compartments. Some full model dendritic compartments were more electrotonically distal than any compartment in the unbranched reduced models. These synapses were shifted to the most distal compartment in the unbranched reduced model; shifting synapses in this way did not cause a large shift in the average synapse position for the unbranched reduced models (Table 1).

Cluster distributions for synchronous excitation To study the effect of synchronous synaptic input, excitatory synapses were grouped into clusters according to their approximate electrotonic distances from the soma. Each cluster contained 13 compartments (from different branches), and two of eight total clusters resided at each of the following approximate distances: $0.175 \mathrm{~L}, 0.35 \mathrm{~L}, 0.525 \mathrm{~L}$ and $0.7 \mathrm{~L}$. In order to be able to directly compare synchronous and asynchronous activation, clusters were activated using 
Table 1 Comparison of basic morphological properties between the full and reduced models. All models contained 3 major branches and possessed the same total surface area. Furthermore, the full and branched reduced models had identical soma to tip L for all branches. The unbranched reduced models were identical to each other except for the lengthwise divisions of their dendritic compartments. Note that mean compartment length (L) and mean compartment surface area

\begin{tabular}{|c|c|c|c|c|c|c|c|c|}
\hline Property & Full & $41 \mathrm{Br}$ & $59 \mathrm{Br}$ & $93 \mathrm{Br}$ & $5 \mathrm{UnBr}$ & $14 \mathrm{UnBr}$ & $50 \mathrm{UnBr}$ & $98 \mathrm{UnBr}$ \\
\hline \# dend comps & 511 & 39 & 57 & 91 & 3 & 12 & 48 & 96 \\
\hline Mean comp length (L) & 0.013 & 0.175 & 0.12 & 0.075 & 0.66 & 0.165 & 0.041 & 0.021 \\
\hline Mean comp SA $\left(\mu \mathrm{m}^{2}\right)$ & 14.5 & 190 & 130 & 81.4 & 2468 & 617 & 154 & 38.6 \\
\hline Mean synaptic position (L) & 0.512 & 0.734 & 0.577 & 0.537 & 0.733 & 0.548 & 0.497 & 0.488 \\
\hline Median $\mathrm{Ra} / \mathrm{SA}\left(\mathrm{M} \Omega / \mu \mathrm{m}^{2}\right)$ & 1.752 & 1.402 & 1.652 & 2.055 & 0.143 & 0.143 & 0.143 & 0.143 \\
\hline
\end{tabular}

common event timetables which maintained the average asynchronous activation rates of their synapses. To allow for direct comparisons with full model responses, cluster positions were directly mapped to their corresponding locations in the branched reduced models. In contrast, cluster positions could not be directly mapped into the unbranched reduced models due to the loss of the detailed branching structure. However, clusters in the unbranched reduced models did maintain major branch locations and approximate electrotonic positions. Furthermore, clusters in the unbranched reduced models included the number of compartments that most closely approximated the TSA of that cluster's compartments in the full model.

\subsection{Simulations}

Simulations were performed using GENESIS 2.3 (http:// www.genesis-sim.org/GENESIS/) on Linux workstations. Simulation data were analyzed in Matlab (The Mathworks, Inc, Natick, MA).

\subsection{Statistics}

For statistical analyses, data set normality was assessed with the Lilliefors test, which showed that all of our sampled data sets were not normally distributed. The non-parametric Mann-Whitney $U$ test was therefore used for statistical comparisons. To correct for multiple simultaneous comparisons, the Mann-Whitney $U$ test significance level was decreased from $\alpha=0.05$ using the Bonferroni correction. All statistical tests were performed in Matlab.

\subsection{Data sharing}

Upon publication, all models along with all current injection and synaptic input patterns used in this study will
(SA) decreased as model compartments were divided. Similarly, divided compartments allowed the mean synaptic position (L) to approximate the full model value in both types of reduced model. Regardless of compartment division, the unbranched reduced models possessed much smaller median ratios of compartment $\mathrm{Ra}$ to SA (median $\mathrm{Ra} / \mathrm{SA}$ ) than either the full or branched reduced models

be made available on the ModelDB public database (http:// senselab.med.yale.edu/ModelDB/default.asp).

\section{Results}

\subsection{Matching passive somatic and dendritic responses}

The passive properties of a neuron model strongly influence its responses in the presence of active conductances (Rall et al. 1992). Therefore, we compared the passive responses between the full model and our branched or unbranched reduced models with different numbers of compartments (Fig. 2). Both $0 \mathrm{~Hz}$ (DC) and 1,000 Hz current injections were applied because the passive response properties of a neuron model depend on the frequency of inputs (Johnston and Wu 1995). Despite the use of the same soma and axon compartments for all models as well as the preservation of certain dendritic morphological properties (see Methods), passive responses to somatic current injection were affected by dendritic properties due to axial current flow and varied by model. Mismatches between the somatic input resistance $\left(\mathrm{R}_{\mathrm{IN}}\right)$ of the reduced and full models ranged from $1 \%$ for the 98 comp unbranched model to $23 \%$ for the 5 comp unbranched model (Fig. 2(a)). In contrast, the $1,000 \mathrm{~Hz}$ input impedance $\left(Z_{\mathrm{IN}}\right)$ was a more challenging measure for reduced models with few compartments: the mismatches between reduced and full model somatic $\mathrm{Z}_{\mathrm{IN}}$ ranged from only $2 \%$ for the 98 comp unbranched model to $67 \%$ for the 5 comp unbranched model (Fig. 2(d)). The quality of the match was greater for reduced models with mean compartment lengths which were more similar to that of the full model, whether or not the branching structure was preserved (Table 1, Fig. 2(a)). The increased $R_{I N}$ and $Z_{I N}$ of the models with the largest trunk compartments near the soma (5comp unbranched and 41 comp branched models) is explained by the decreased axial current that can exit the soma because of the high axial resistance $\left(\mathrm{R}_{\mathrm{A}}\right)$ of the first 

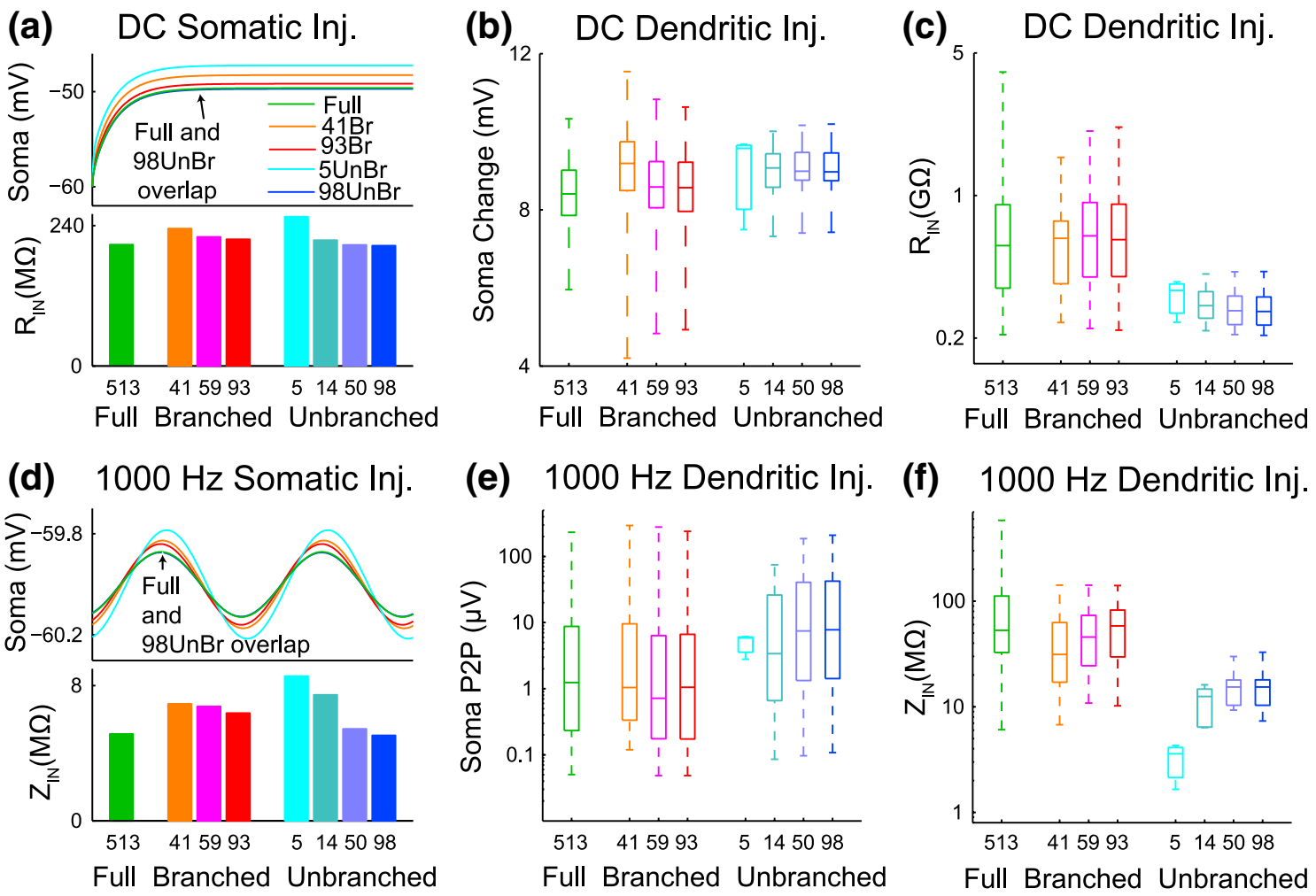

Fig. 2 Passive somatic responses to DC or $1,000 \mathrm{~Hz}$ current injection into the soma or individual dendritic compartments. For panels C, E and $\mathrm{F}$, a $\log$ scale was used for the $\mathrm{y}$-axis to facilitate comparisons between models. (a) Voltage deflections (top, selected models) and $\mathrm{R}_{\mathrm{IN}}$ (bottom, all models) are plotted for $50 \mathrm{pA}$ current injections into the soma. $\mathrm{R}_{\mathrm{IN}}$ was calculated by dividing the steady state voltage change by the DC amplitude. Note the slight increase in $\mathrm{R}_{\mathrm{IN}}$ for the most reduced branched or unbranched models. (b) Each dendritic compartment was separately injected with $50 \mathrm{pA}$ of DC. The box and whisker plots show the amplitudes of the resulting somatic voltage deflections: the middle horizontal bar represents the median while the upper and lower horizontal bars represent the 75th and 25th percentiles, respectively; the whiskers identify the most extreme values found. Box and whisker plots, bar graphs, and raster plots

dendritic compartment, which lumps together the $\mathrm{R}_{\mathrm{A}}$ of several compartments in the full model. Therefore the dendritic trunk is charged less and the soma more by somatic current injection. This effect is larger for $1,000 \mathrm{~Hz}$ input to the soma (Fig. 2(d)), and thus fast somatic currents such as those during an action potential (AP) can be expected to create larger somatic voltage deflections with a different time course in models with high $\mathrm{R}_{\mathrm{A}}$ values in the initial trunk compartments. This dependence of fast transients on all axial currents in a neuron should be kept in mind, for example when examining the interaction between ion channel kinetics and morphology to produce smooth or kinky action potential onsets, an issue that has recently gathered much interest (McCormick et al. 2007; Naundorf et al. 2006).

Next we examined the reproduction of somatic responses to dendritic input in our reduced models. We found that the present the models in the same order consistently throughout the paper. Note that the median somatic voltage change for injection into different dendritic compartments was comparable for all models, but that branched reduced models showed slightly larger variability in somatic responses than the full model, whereas unbranched reduced models showed slightly lower variability. (c) Distributions of local dendritic $\mathrm{R}_{\mathrm{IN}}$ values for each model. The median and quartile values but not whisker extents were similar between the branched reduced models and the full model, whereas the unbranched reduced models had lower median dendritic $\mathrm{R}_{\mathrm{IN}}$ and showed much less variability between compartments. $(\mathbf{d}-\mathbf{f})$ Same plots as $(\mathbf{a}-\mathbf{c})$, except the injected current was a $1,000 \mathrm{~Hz}$ sinusoidal input with a peak-to-peak (P2P) amplitude of $50 \mathrm{pA}$. Input impedance $\left(\mathrm{Z}_{\mathrm{IN}}\right)$ was calculated as $\mathrm{P} 2 \mathrm{P}$ voltage deflection divided by $50 \mathrm{pA}$

match to the median passive somatic response amplitude of the full model to dendritic current injections in all compartments (one by one) ranged from $2 \%$ for the $93 \mathrm{comp}$ branched model to $14 \%$ for the 5 comp unbranched model (Fig. 2(b)). In contrast, with $1,000 \mathrm{~Hz}$ injection, we found that median response amplitude of the closest matching branched reduced model (93comp) was $15 \%$ smaller than that of the full model while the closest matching unbranched reduced model's median response amplitude was $174 \%$ larger than that of the full model (Fig. 2(e)). This higher median response amplitude is due to the fact that many fewer compartments are located at distal positions in the unbranched models.

In contrast to the small differences between the somatic passive responses of the full, branched and unbranched reduced models to dendritic input, the local dendritic responses diverged between branched and unbranched 
model reductions. Indeed, mismatches between the median local dendritic $\mathrm{DC} \mathrm{R}_{\mathrm{IN}}$ of the branched reduced and full models ranged from only $7 \%$ to $12 \%$ while mismatches for the unbranched reduced models ranged from $40 \%$ to $52 \%$ (Fig. 2(c)). For the more challenging measure of median local dendritic $Z_{\mathrm{IN}}$ with $1,000 \mathrm{~Hz}$ input, the mismatches between the branched reduced and full models ranged from only $10 \%$ to $41 \%$ while unbranched reduced model mismatches ranged from $71 \%$ to $93 \%$ (Fig. 2(f)). The unbranched reduced models possessed lumped branches which were thicker than those of the full or branched reduced models and therefore exhibited smaller local $R_{I N} S$ and $Z_{\mathrm{IN}}$ because their dendritic compartments possessed much smaller median $\mathrm{R}_{\mathrm{A}}$ relative to surface area (Table 1). Due to this unavoidable characteristic of unbranched models, current flowed away from the injection site more easily and did not charge the membrane locally as much as in the full or branched reduced models. This difference between branched and unbranched models could be quite important for modeling studies of excitatory postsynaptic potential (EPSP) amplification, for instance, because the amount of amplification depends non-linearly on the dendritic voltage reached (Golding and Spruston 1998).

Despite matching the median dendritic $\mathrm{R}_{\mathrm{IN}}$ and $\mathrm{Z}_{\mathrm{IN}}$ of the full model, the branched reduced models lacked dendritic $R_{I N} S$ or $Z_{I N} S$ as large as some present in the full model. Indeed, the full model possessed maximum dendritic $D C R_{I N} S$ and $1,000 \mathrm{~Hz} Z_{I N} S$ which were respectively about twice and four times as large as any possessed by a branched reduced model (box plot whiskers in Fig. 2(c,f)). Furthermore, the full model possessed 105 compartments which had larger 1,000 Hz $\mathrm{Z}_{\mathrm{IN}} \mathrm{S}$ than any exhibited by a branched reduced model compartment. The unbranched reduced models showed even less variability in local dendritic $R_{I N} S$ and $Z_{I N} S$, due to their lack of small side branches.

In conclusion, the unbranched reduced models matched the full model's passive somatic responses but failed to match its dendritic $R_{I N} S$ and $Z_{I N} S$. In contrast, the branched reduced models matched both the somatic and median dendritic passive responses of the full model to current injection. However, the branched reduced models lacked a population of dendritic $\mathrm{R}_{\mathrm{IN}} \mathrm{S}$ or $\mathrm{Z}_{\mathrm{IN}} \mathrm{S}$ as large as many present in the full model. These similarities and differences in passive properties between the reduced and full models provide much of the basis for emerging similarities and differences in the active models.

3.2 Using 'mock' action current injection to predict differences in AP size

The detailed spike shape is a frequently studied electrophysiological feature (Bean 2007) which can depend on a neuron's morphology (McCormick et al. 2007). It is therefore an interesting question whether reduced computer models should be expected to faithfully reproduce this feature of the experimental data. The results shown above suggest that purely passive differences in axial current flow between full and reduced models could confound studies of how active conductances determine the detailed spike shape. Unfortunately, isolating differences in spike shape due to axial current flow in the presence of voltage-gated conductances is complicated due to the non-linear interactions between the membrane voltage trajectory and conductance activation. The injection of a fixed 'mock AP current' into the passive models allowed us to determine which differences in the active AP should be expected based on passive properties alone. For a mock AP, we used a square current pulse injection with $5 \mathrm{nA}$ amplitude and $0.5 \mathrm{~ms}$ duration, which produced a voltage spike with an amplitude and rise time similar to a $\mathrm{Na}^{+}$action potential. We found that the mock AP voltage deflection was within $1 \%$ of the full model's value for the 98 comp unbranched model; however, as the average electrotonic size of compartments increased, the closeness of reduced model voltage deflections to that of the full model decreased (Table 1, Fig. 3(a)). Indeed, the mock AP voltage deflection showed a steeper onset and was 55\% larger in the 5 comp unbranched model than in the full model due to the reduced axial currents flowing into the high Ra dendritic trunk of the 5comp model (Fig. 3(b)). For the same reason, the mock action current led to a much decreased voltage deflection in the dendrites of this model (Fig. 3(c)). Note that the same factors contributed to dendritic mock AP size differences between the 14comp unbranched model and full model, and that even the branched reduced models showed smaller dendritic mock AP sizes due to larger Ra values of the trunk dendritic compartments. Overall, our results with mock AP current injection show that large dendritic trunk compartments can lead to differences in AP size. Additionally, decreased passive back propagation of the AP could influence the activation of dendritic voltage gated currents and therefore could further exacerbate differences in integrative properties for the most reduced models. Nevertheless, both branched reduced models and unbranched models with $>10$ compartments generally exhibited close matches to full model somatic mock AP size.

\subsection{Analysis of detailed somatic shape of sodium APs for different levels of model reduction}

After isolating the influence of reduced model architectures on voltage transients for fixed current injection pulses, we added the full complement of ion channels back in to compare $\mathrm{Na}^{+}$AP shapes between the full and reduced models. This set of simulations allowed us to determine the 
(a) Somatic response

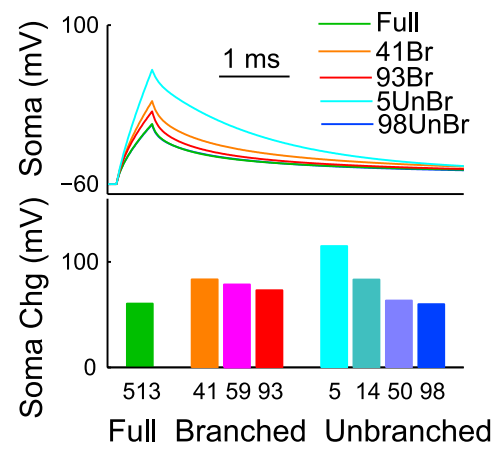

(b) $I_{\text {axial }}$ into dendrites

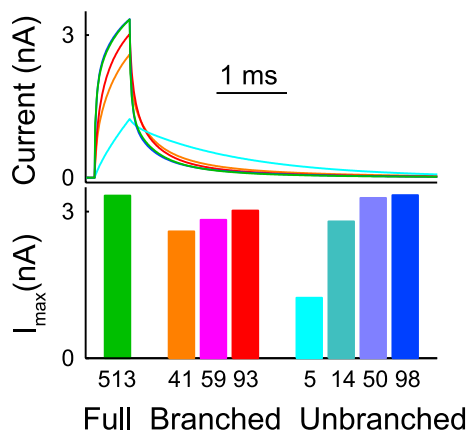

(c) Avg prox response

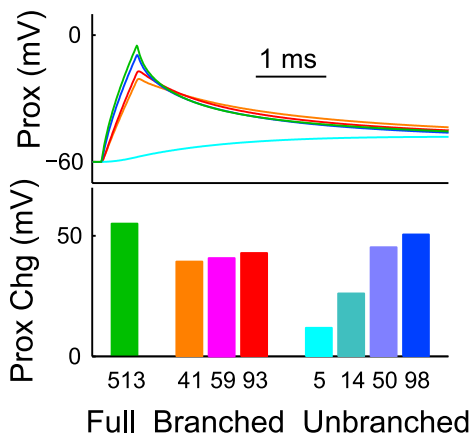

Fig. 3 The passive somatic response amplitude to a mock action current depended on dendritic axial resistance. The stimulus was a brief $(0.5 \mathrm{~ms})$ large $(5 \mathrm{nA})$ somatic current pulse which caused a voltage response in the passive soma that mimicked the amplitude and rise-time of an AP in the absence of voltage gated conductances (mock AP). (a) The mock AP was smallest in the full model and the most subdivided (50comp and 98comp) unbranched reduced models.

influence of the described passive mismatches on voltagegated channel activation, which could further exacerbate spike shape mismatches.

Using the same active conductance parameter set that was shown to reproduce physiological GP neuron activity in the full model (see Methods) for all models, the active full and reduced models each exhibited tonic, regular spontaneous spiking (Fig. 4(a)). Spikes were always initiated in the axon compartment due to its high $\mathrm{NaF}$ conductance. Despite identical conductance densities, spike shapes differed somewhat depending on each model's morphology. As predicted by the mock AP comparisons, the somatic spikes of the 5comp model were taller than those of the full model (by $7.9 \mathrm{mV}$ ). Spikes in the other reduced models were also taller than those in the full model, but not by as much, and they followed the same pattern as for the height of the mock AP (Fig. 4(b) left inset). It should be kept in mind, however, that the amount of variability in spike height between the full and reduced models was not large relative to the variability seen between different GP cells in vitro (standard deviation= $10.8 \mathrm{mV}$ ) (Gunay et al. 2008). Note that AP height depended on the average dendritic compartment length rather than on the preservation of the detailed dendritic branching structure (see Table 1). Spike height differences were due to variations in the amount of axial current leaving the soma during a spike (Fig. 4(d), left inset): models with less axial current exiting the soma during a spike (Fig. 4(d), compare positive peaks) possessed taller action potentials (Fig. 4(b), left inset) because more current remained in the soma to charge the somatic membrane. Models with less axial current flow also exhibited slightly more hyperpolarized spike initiation thresholds (Fig. 4(c), left inset) because less positive driving current was able to
The ability of the reduced models to match the full model mock AP response improved as compartments were divided. (b) Models with more finely-divided compartments allowed larger axial currents to exit the soma into the dendrites during the mock AP. (c) Models with larger axial currents exiting the soma exhibited larger average voltage deflections in their most proximal dendritic compartments (those directly connected to the soma)

exit the soma into the dendrites during spike onset. The medium dendritic gNa level in our default full model was too low to propagate active dendritic APs, but a parameter set with high dendritic gNa allowing active dendritic spiking showed quite similar results for the dependence of spontaneous spike shape on model reduction (Supplemental Fig. S1, Online Resource 1), indicating that these findings generalize to spiking and non-spiking dendrites.

A direct consequence of smaller axial current flows into the dendrites during a mock AP was less depolarization of the dendrites (Fig. 3(c), passive case). Less depolarized proximal dendrites contributed less positive axial current back to the soma immediately following the spike during the fast afterhyperpolarization (fAHP) (Fig. 4(d), right inset). Indeed, fAHPs were deeper in models with smaller axial current flows back into the soma immediately following a spike (e.g. the 5comp model, Fig. 4(b) right inset, Fig. 4(d) inset). In contrast to the small spike height differences, the difference between the full and 5 comp model fAHPs was $17.3 \mathrm{mV}$, about six times larger than the experimental variability for this measure (Gunay et al. 2008). Deeper fAHPs in the 5 comp model lasted for many milliseconds following a spike (Fig. 4(c), right inset). In sum, axial current flow was a key determinant of spike shape differences: the reduced models that exhibited axial current flows most similar to those of the full model (e.g. the 50 and 98 comp unbranched models) also provided the closest matches to the full model's spike shape as measured by the mean absolute error (MAE, defined as the mean point by point absolute value difference between reduced and full model traces) (Fig. 4(e)). Branched model MAEs were large relative to those of the unbranched models with similar numbers of compartments. This was due to the fact that branched 
(a) Spontaneous spiking

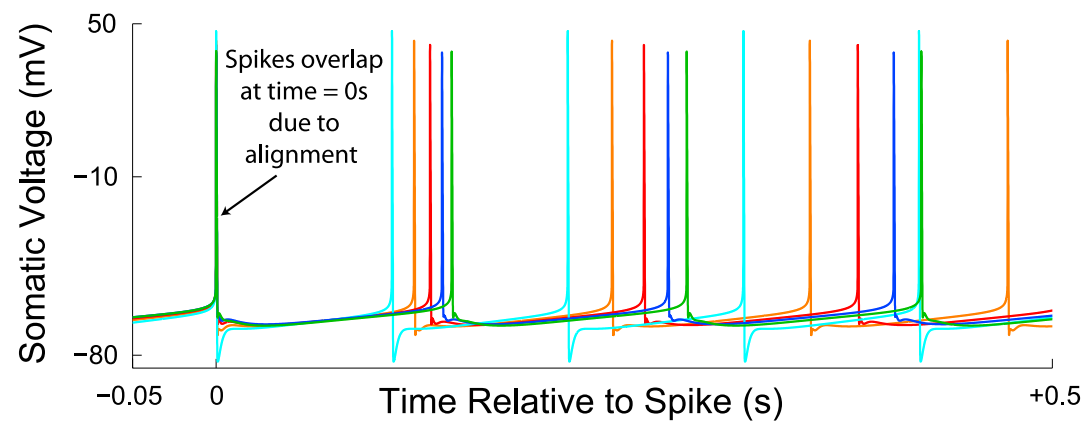

(b) Spont. spike shape

(c) Spont. spike shape
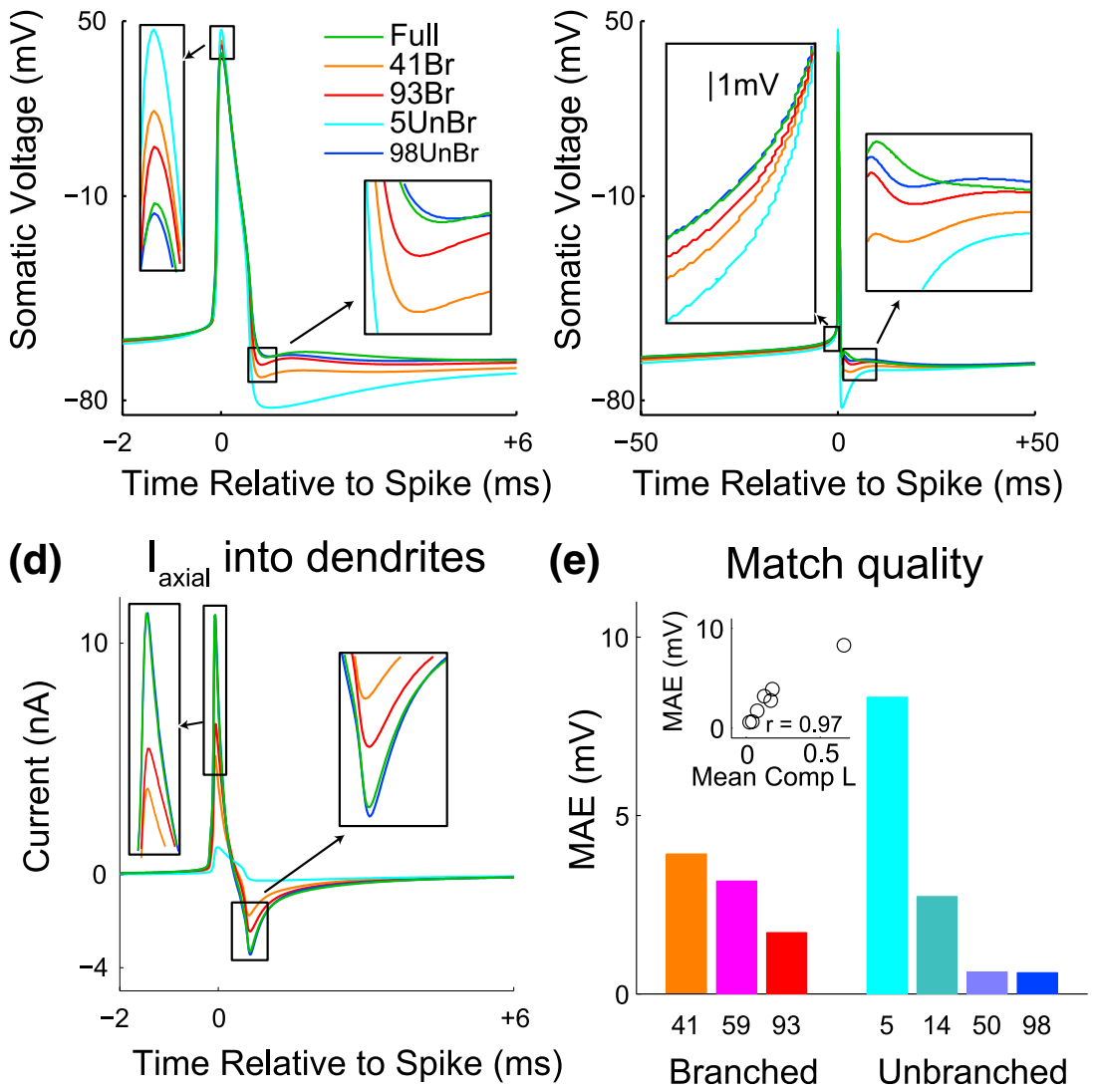

Fig. 4 Shape analysis of spontaneous somatic APs in fully active models. Models at all levels of reduction possessed identical somatic, axonal and dendritic conductance densities. (a) Spontaneous spiking is shown for the full and reduced models. (b) The relative spike heights in the active models (left inset) corresponded directly to the relative mock AP response amplitudes shown in Fig. 3(a). Models with shorter spike heights possessed shallower fast afterhyperpolarizations (fAHPs) as well (right inset). (c) Expanded time scale shows steeper rise time to spike onset in the most reduced models (left inset) and extended differences in fAHP potential (right inset). (d) Axial current between soma and dendritic trunk compartments. The spike depolarization led to a large positive current into the dendrites; somatic spike size was increased in

reduced model trunk dendritic compartments were still electrotonically longer than those of the unbranched models since a large number of compartments was necessary simply to reflect the entire branching structure

models with less axial current due to high Ra values. During the somatic fAHP, the axial current reversed, and current flowing back into the soma from the depolarized dendrites led to a decrease in fAHP amplitude. Therefore fAHP was largest in the most reduced models because they had the highest Ra values into the dendritic trunk compartments directly connected to the soma. (e) The quality of the match to the full model spontaneous spike shape shown in (b) was calculated for each model as the MAE of the voltage traces from $2 \mathrm{~ms}$ before to $6 \mathrm{~ms}$ after the spike peak. To calculate MAE, spikes were aligned so that they crossed $0 \mathrm{mV}$ at the same time. MAE was highly correlated with mean compartment electrotonic length ('mean comp L'; $r=0.97$, Pearson correlation coefficient; see inset)

of the full model (see Table 1). Indeed, we observed a strong positive correlation $(r=0.97$, Pearson correlation coefficient) between spike shape MAE and mean compartment electrotonic length (Fig. 4(e), inset). 
We considered the possibility that manipulation of conductance densities in the 5comp model could allow a closer match to the full model's spike shape. The full model's spike was shorter (by $7.9 \mathrm{mV}$ ) and its fAHP shallower (by $17.3 \mathrm{mV}$ ) than that of the 5comp model. By hand-tuning the 5comp model's conductance densities, these mismatches in spike height and fAHP depth respectively could be decreased by $97 \%$ and $77 \%$. This level of error reduction was achieved through a 4-fold decrease in the somatic and axonal $\mathrm{NaF}$ conductance densities and a 16-fold decrease in Kv2, Kv3, and Kv4fast/slow. While our hand-tuning allowed a much closer match of spike height and fAHP depth, these conductance density changes also caused the spike width at $-20 \mathrm{mV}$ to increase from $0.53 \mathrm{~ms}$ to $0.88 \mathrm{~ms}$ (the full model spike width was $0.58 \mathrm{~ms}$ ). Because spike width was a key determinant of calcium entry through HVA channels in the model, increasing the spike width lead to an increase in SK channel activation that secondarily altered multiple model properties. Overall, hand-tuning conductance densities in reduced models away from the full model parameter set always produced tradeoffs in our experience, and it was not possible to fully compensate for mismatches incurred by morphological differences. In addition, the channel densities that made the 5comp model spike shapes most similar to electrophysiological recordings were several-fold different from the original channel densities in the full morphology, and thus the biological interpretation of such highly reduced models with channel densities adjusted to match physiological recordings would need to take this fact into consideration.

\subsection{Analysis of back propagating APs (bAPs) at different levels of model reduction}

A neuron model's bAP amplitudes have important consequences for the modeling of spike time dependent synaptic plasticity (Caporale and Dan 2008). If a reduced model's bAP amplitudes were appreciably different from those in a full model, coincident bAPs and EPSPs in the dendrites would fail to evoke similar local responses. To examine the effect of model reduction on bAP amplitudes during fast spiking activity as may be expected for GP neurons in vivo, $75 \mathrm{~Hz}$ spiking was elicited in each model by somatic DC injection. We normalized bAP amplitudes to somatic AP height so that models with taller somatic spikes did not mistakenly appear to have more effective back propagation. In most dendritic compartments of the full and reduced models, bAP amplitudes decayed at about the same rate with electrotonic distance from the soma (Fig. 5(a)). However, in the most highly reduced models even the trunk dendritic compartments were at a considerable electrotonic distance from the soma (Fig. 5(a)), and therefore the initial decrease in bAP amplitude was much more pronounced. A further difference between the reduced and full models was that variability in bAP amplitude for different dendritic branches at a given electrotonic distance from the soma was much larger in the full model. In particular, one full model branch had a greatly increased bAP amplitude (Fig. 5( $\left.a_{1}\right)$, black arrow). Indeed, the bAP amplitude at the end of this branch $(L \approx 0.5)$ was $49 \%$ of the somatic AP height, while the largest bAP amplitudes observed at this electrotonic position in any branched or unbranched reduced models were respectively only $13 \%$ or $16 \%$ of somatic AP height. This large bAP disappeared in the full model without dendritic gNa (Supplemental Fig. S2A, Online Resource 1), which meant that the large amplitude was due to local amplification by $\mathrm{gNa}$. Thus even when the branching structure of the full morphology was maintained, morphological reduction still resulted in significant functional changes in the presence of active conductances.

The primary cause of bAPs lies in the propagation of axial current. The peak axial current during a bAP quickly decayed with electrotonic distance at about the same rate in the full and reduced models (Fig. 5(b)) such that only a small fraction of the initial axial current at the soma boundary even reached intermediate dendritic positions at $0.5 \mathrm{~L}$ (Fig. $\left.5\left(b_{1}\right)\right)$. The peak axial current reaching distal locations (e.g. $1.0 \mathrm{~L}$ ) was quite variable between dendritic branches (Fig. 5(c)) though the variability was diminished by either removing dendritic $\mathrm{gNa}$ or setting it to a high density that supported active spike back propagation (Supplemental Fig. S2, Online Resource 1). However, the peak axial current reaching any given compartment did not determine the local bAP amplitude. Instead, the amplitude was primarily determined by the local $\mathrm{Z}_{\mathrm{IN}}$ at each location. For example, in the full model at $0.54 \mathrm{~L}$, the branch with the largest bAP amplitude $\left(1,000 \mathrm{~Hz} \mathrm{Z}_{\mathrm{IN}}\right.$ of $\left.304 \mathrm{M} \Omega\right)$ received only $35 \%$ of the axial current received by the branch with the smallest bAP $\left(1,000 \mathrm{~Hz} \mathrm{Z}_{\mathrm{IN}}\right.$ of $\left.76 \mathrm{M} \Omega\right)$. Such a large spread in $1,000 \mathrm{~Hz} Z_{\mathrm{IN}}$ was not observed in any of the reduced models, explaining the smaller spread of bAP amplitudes (Fig. 5(a)). While the branched reduced models possessed the same branches as in the full model, their $1,000 \mathrm{~Hz} \mathrm{Z}_{\mathrm{IN}} \mathrm{S}$ were smaller due to their larger compartment sizes, leading to the observed mismatches in bAP amplitudes. These differences between the full model, branched reduced models and unbranched reduced models should be considered when using modeling to study bAPs or employing them to study spike dependent plasticity rules.

3.5 Effects of model reduction on the somatic spike frequency vs. current Injection (fI) curves

The somatic fI curve is a basic neuronal input-output function which is frequently recorded by cellular electrophysiologists due to the important information that it yields about neuronal excitability. Possible mismatches in the somatic fI curve due 
$\left(a_{1}\right) \quad$ Full model $\quad\left(a_{2}\right)$ Branched models $\left(a_{3}\right)$ Unbranched models
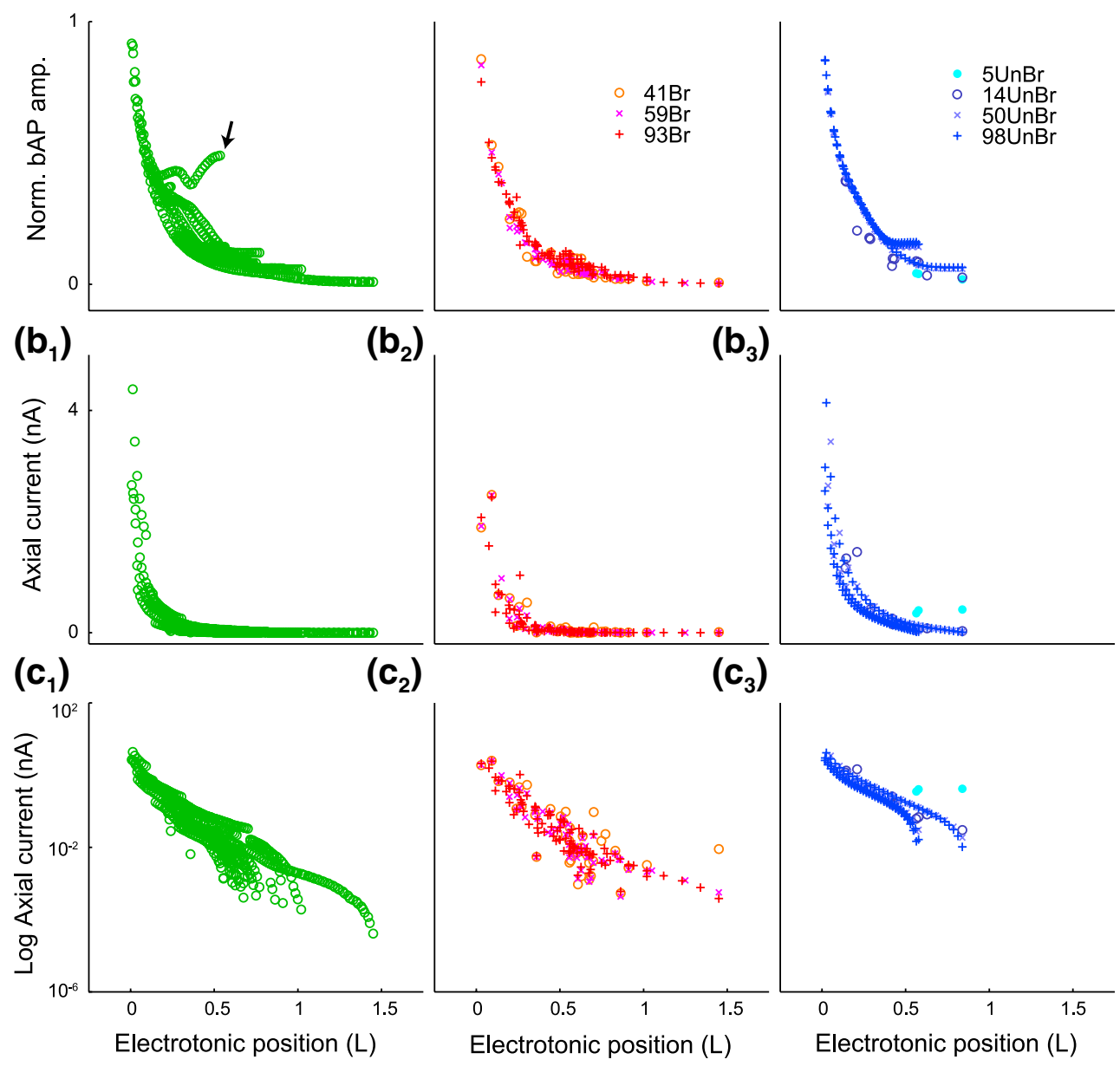

Fig. 5 Back propagating AP (bAP) amplitudes can be much larger in the full model than in the reduced models due to larger local dendritic high frequency $Z_{\mathrm{IN}} \mathrm{s}$. All models were driven to fire at $75 \mathrm{~Hz}+-0.1 \%$ with DC somatic injection. (a) The bAP amplitude in each compartment was normalized to the soma spike amplitude and plotted against the electrotonic distance from the soma for the full model $\left(a_{1}\right)$, branched models $\left(a_{2}\right)$ and unbranched models $\left(a_{3}\right)$. Most models showed a similar decay in bAP amplitude with electrotonic distance. However, the 5comp and 14comp unbranched models showed much smaller bAP sizes in their proximal dendrites because these compart-

to model reduction could result in consequential changes with respect to excitability in the presence of synaptic input. To assess the extent of such mismatches, we compared somatic fI curves between the full model and the branched or unbranched reduced models (Fig. 6(a)). All models exhibited spontaneous spiking which could be eliminated by injecting between $-31 \mathrm{pA}$ and $-41 \mathrm{pA}$ of DC into the soma. For positive current injection amplitudes between $0 \mathrm{pA}$ and $500 \mathrm{pA}$, the full and reduced models exhibited nearly linear increases in spike rate. However, the somatic fI curve was steeper in some of the reduced models than in the full model. This slope mismatch was related to differences in somatic $\mathrm{R}_{\mathrm{IN}}$ between the full and reduced models (compare Figs. 2(a) ments were already electrotonically quite distant from the soma. In addition, the full model showed more variable bAP amplitudes in different branches (see black arrow) than even the most detailed branched reduced models. (b) The maximum amount of axial current flowing into each compartment during a spike (referred to simply as axial current) is plotted against electrotonic distance from the soma. (c) Log scale representation of the plots shown in (b). Note that the decay of axial current with electrotonic distance is approximately loglinear and is quite similar between the full and reduced models

with 6(a)). Models with larger somatic $\mathrm{R}_{\mathrm{IN}}$ allowed more somatically-injected current to charge the somatic and axonal membranes instead of flowing into the dendrites. This difference was most pronounced for the 5comp unbranched model, since this model had by far the highest somatic $\mathrm{R}_{\mathrm{IN}}$ (Fig. 2(a)). Overall, the differences in spike rate between the full and reduced models were small (e.g. spontaneous rate varied by only $2.4 \mathrm{~Hz}$ ) relative to the variability that is observed between different GP cells recorded in vitro (standard deviation for spontaneous rate $=5.9 \mathrm{~Hz}$ ) (Gunay et al. 2008). Therefore our data indicate that somatic fI curve mismatches are not one of the important limitations of reduced models, with the possible exception of highly 
(a) Somatic fl curve

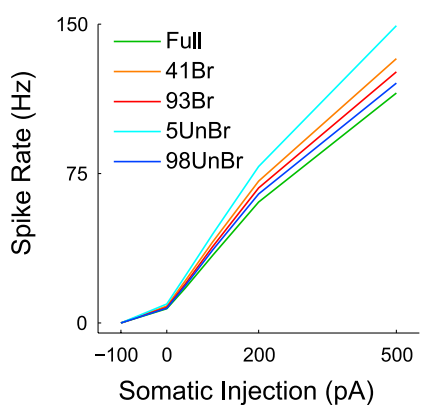

(b) Dendritic fl curve

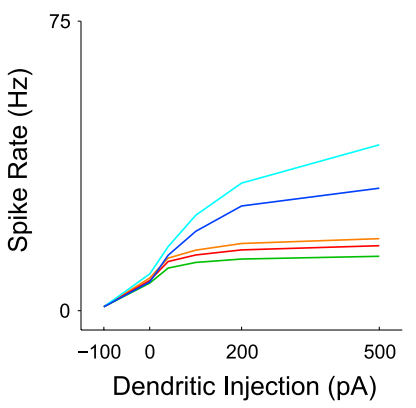

(c)

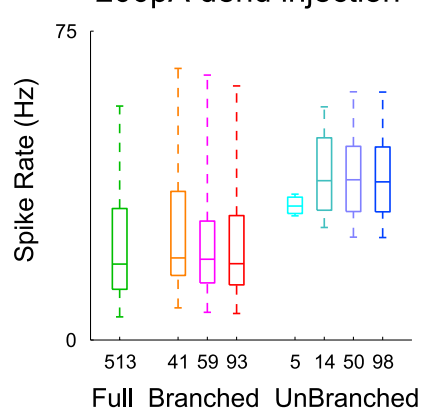

(d) C, w/Passive Dendrites

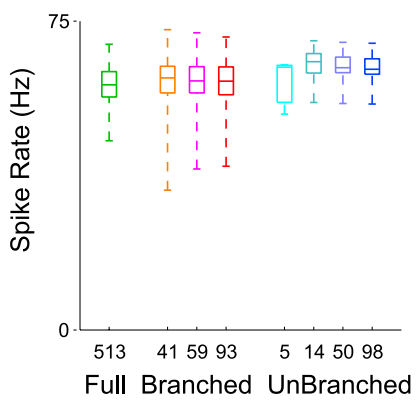

Fig. 6 The spike frequency response to somatic and dendritic DC injection (fI curve) at different levels of model reduction. DC injection amplitudes ranged from $-100 \mathrm{pA}$ to $+500 \mathrm{pA}$ to elicit the full physiological range of spike rates. (a) fI curve of each model for somatic current injections. Models with higher somatic $\mathrm{R}_{\mathrm{IN}} \mathrm{S}$ (see Fig. 2(a)) exhibited steeper somatic fI curves. Note that subdividing the compartments of the reduced models allowed closer matches to the somatic fI curve of the full model. (b) fI curve of each model for DC injection into a sample dendritic compartment at an electrotonic distance of $0.83 \mathrm{~L}$ from the soma. The injected compartment was positioned on the same sub-branch in the full and branched reduced models and on the same major branch in the unbranched reduced models. Note that the branched reduced models closely matched the

reduced models with severely limited axial currents such as our 5comp model.

We again considered the possibility that the somatic fI curve of the 5comp model could be improved relative to that of the full model by hand-tuning its conductance densities. Indeed, by decreasing axonal $\mathrm{NaP}$ by $12.5 \%$ and increasing somatic SK by $50 \%$, the 5 comp model's somatic fI curve closely matched that of the full model. However, these manipulations did not appreciably decrease the discrepancy between the $5 \mathrm{comp}$ model's spike rate responses to dendritic current injection relative to those of the full model: this discrepancy will be addressed in the next section.

\subsection{Effects of model reduction on fI curves for dendritic current injection}

While the somatic fI curve is an important measure of the excitability of a neuron, it does not take into account input processing taking place in active dendrites. Active dendrites are capable of many types of input processing, depending on their precise morphology and their complement of ion channels (London and Hausser 2005). As a first step towards characterizing the influence of model reduction on dendritic processing, we recorded the spike frequency with different dendritic DC injection amplitudes at a relatively distal location $(\sim 0.8 \lambda)$ in each model. As shown in Fig. 6(b), the dendritic fI curves were much steeper in the unbranched reduced models than in the full or branched reduced models. To test whether mismatches in dendritic fI

dendritic fI curve of the full model while the unbranched reduced models did not provide close matches. (c) Box plots for distributions of spike frequency responses for $200 \mathrm{pA}$ DC injections into each dendritic compartment of each model. The median spike frequency response to dendritic injection was much higher in the unbranched reduced models than in either the full model or the branched reduced models. The minimum spike frequency response to dendritic injection was also much higher in the unbranched reduced models than in either the full model or the branched reduced models. (d) Same as (c) except that all channels were removed from the dendrites. Note the much closer match between all models and the strong correspondence to the relationships shown in Fig. 2(b)

curves depended on the particular dendritic location chosen, we injected $200 \mathrm{pA}$ of DC into every dendritic compartment of each model and compared the distribution of spike frequencies between models (Fig. 6(c)). For all dendritic compartments, the median spike frequencies of the unbranched reduced models were about twice as large as those in the full or branched reduced models (Fig. 6(c)). In addition, the minimum spike frequency was much larger in the unbranched reduced models than in the full or branched reduced models (Fig. 6(c)). However, this difference was sensitive to the dendritic $\mathrm{gNa}$ level: with high dendritic gNa, the full model responded to dendritic current injection with high spike frequencies that matched the unbranched models, although the spread of spike frequency responses remained much higher in the full model (Supplemental Fig. S4C, Online Resource 1). Overall, these results showed that differences between full and reduced models in their dendritic fI curves were quite large, and furthermore depended on the complement of ion channels.

Next, we sought to understand more fully the relationship between dendritic current injection and somatic spike rate. To examine the possibility that different spike frequency responses were simply related to the electrotonic distance of each injected compartment to the soma, we compared the spike rate response during $200 \mathrm{pA}$ DC dendritic injection to the electrotonic position of each injected dendritic compartment. Perhaps surprisingly, there was considerable variability between spike rate responses to current injections at matching electrotonic positions in 
different branches of each model (Fig. 7(a)). Because electrotonic position need not be directly correlated to local $\mathrm{R}_{\mathrm{IN}}$, we hypothesized that local dendritic $\mathrm{R}_{\mathrm{IN}}$ could be a stronger predictor of spike frequency responses. Indeed, we found a very close relationship between high values of local dendritic $\mathrm{R}_{\mathrm{IN}}$ at the site of current injection and low values of somatic spike frequency regardless of model reduction level (Fig. 7(b)). Since the unbranched models lacked compartments with high local $\mathrm{R}_{\mathrm{IN}} \mathrm{S}$ due to their collapsed branching structures (Fig. 2(c)), their minimum spike frequency responses to dendritic current injections were higher (Fig. 6(c)).

To determine whether dendritic conductances were responsible for the differences in spike rates with dendritic DC injection, we removed all dendritic conductances and measured spike rates in response to the same stimuli used for Fig. 6(c). We found that passive dendrites allowed close matches between all models regarding spike rate responses to dendritic DC injections (Fig. 6(d)). The spike rates achieved with current injection into passive dendrites were much higher than those with active dendrites, indicating that activation of outward currents during dendritic depolarization primarily determined slow spike responses. Because dendritic depolarization with input is directly dependent on the local $R_{I N}$, the unavoidable reduction of local dendritic $R_{I N}$ when multiple dendritic branches are collapsed in unbranched models leads to significant mismatches in the response to input when active conductances are present. These mismatches can lead to either decreased or increased spiking based on the relative predominance of inward or outward dendritic currents.
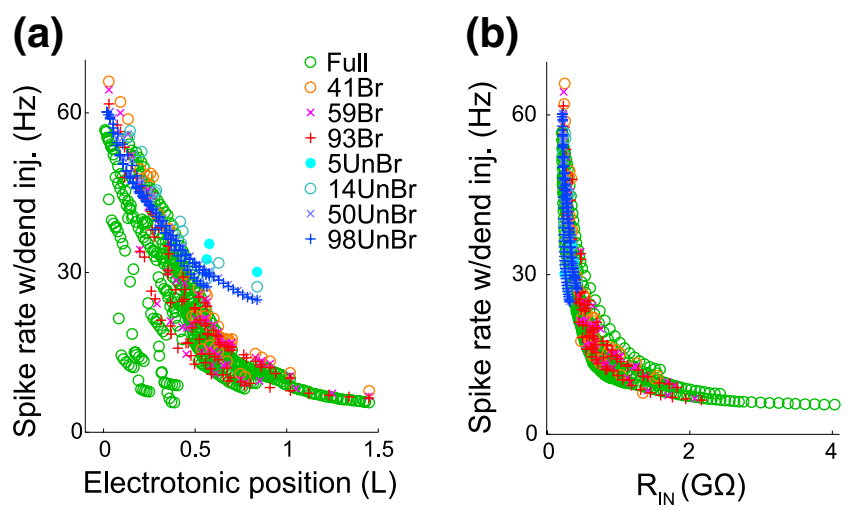

Fig. $7 \mathrm{R}_{\mathrm{IN}}$ was a stronger predictor than electrotonic position of the spike rate response to dendritic injection. The $\mathrm{R}_{\mathrm{IN}}$ of a dendritic compartment was calculated as for Fig. 2. (a) The spike rate response during 200 pA DC injection into each dendritic compartment depended weakly on electrotonic distance from soma (L). (b) In contrast, the spike rate response during $200 \mathrm{pA}$ DC injection into a dendritic compartment depended strongly on the injected compartment's $\mathrm{R}_{\mathrm{IN}}$
3.7 Comparison of spike frequency response to synaptic inputs at a range of input frequencies across models

The integration of distributed synaptic inputs into spiking output is one of the fundamental computational functions of a neuron (Destexhe et al. 2003; Gulledge et al. 2005). Accurate synaptic integration relative to a full model or biological neuron is particularly important for a reduced model that will be incorporated into network simulations. A first order approximation of the input/output function of a neuron model is frequently represented by $\mathrm{fF}$ curves, which compare spike frequency vs. input frequency at different mixes of inhibition and excitation. We generated $\mathrm{fF}$ curves for all models by recording the spike frequencies for increasing rates of asynchronous excitatory input to all dendritic compartments at three levels of inhibition. We applied identical synaptic conductance activation patterns between all models (see Methods). We found that the $\mathrm{fF}$ curves of all reduced models matched the full model more closely than the fI curves for dendritic current injection had suggested (Fig. 8(a), compare to Fig. 6(b,c)). Nevertheless, the unbranched models showed a somewhat larger spike frequency increase with increasing excitatory input rates as suggested by the dendritic fI curves, whereas the 59 comp and 93 comp branched reduced models presented a close approximation to the $\mathrm{fF}$ curves of the full model. As we observed with dendritic fI curves, the degree to which the $\mathrm{fF}$ curves matched between the models was sensitive to the dendritic $\mathrm{gNa}$ level. With high dendritic $\mathrm{gNa}$, no reduced model was able to match the steep $\mathrm{fF}$ curves of the full model due to differences in dendritic spike initiation and propagation (Supplemental Fig. S5, Online Resource 1).

To better understand the $\mathrm{fF}$ curve mismatches of the unbranched models with medium dendritic $\mathrm{gNa}$, we studied the somatic and dendritic voltage fluctuations of the 93 comp, 98comp and full models after removing their somatic and axonal active conductances (Fig. 8(b-d)). The somatic voltages were shifted relative to one another in a manner consistent with the relative firing rates in the $\mathrm{fF}$ curves. However, the magnitude of somatic voltage fluctuations was quite similar between the full and reduced models (Fig. 8(b)). In contrast, the dendritic voltage fluctuations in the unbranched $98 \mathrm{comp}$ model were much smaller than in the full or branched 93comp model at each level of inhibition (Fig. 8(c,d); note the close similarity between the local fluctuations of the full and $93 \mathrm{comp}$ branched models). We hypothesized that these different dendritic voltage fluctuations influenced dendritic conductances in a complex manner and prevented the 98 comp model from precisely matching the $\mathrm{fF}$ curves of the full model. To test this, we removed all dendritic conductances from each model and reproduced the $\mathrm{fF}$ curves. As we predicted, the $\mathrm{fF}$ curves of the full and reduced models 
$\left(a_{1}\right) \quad$ No Inhibition

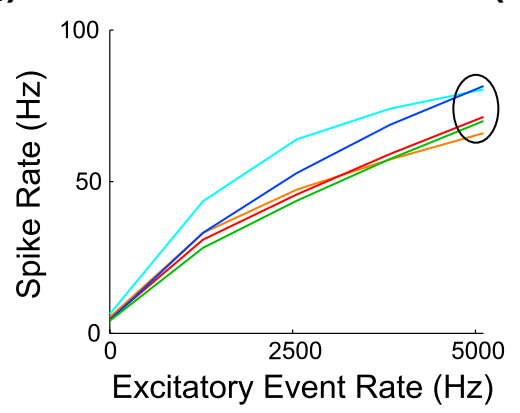

$\left(\mathrm{a}_{2}\right)$ Medium Inhibition

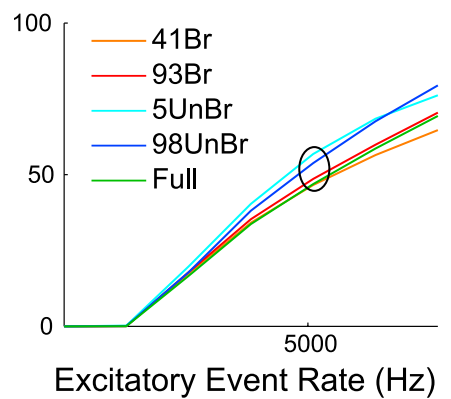

$\left(a_{3}\right)$ High Inhibition

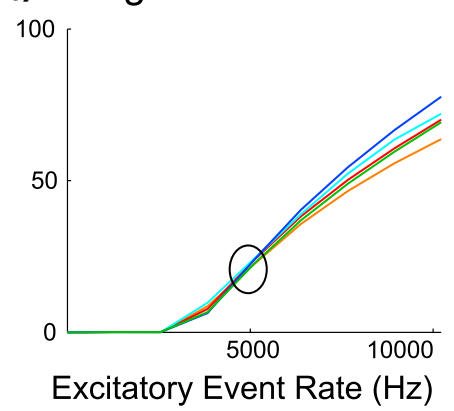

$\left(b_{1}\right)$

$\left(b_{2}\right)$
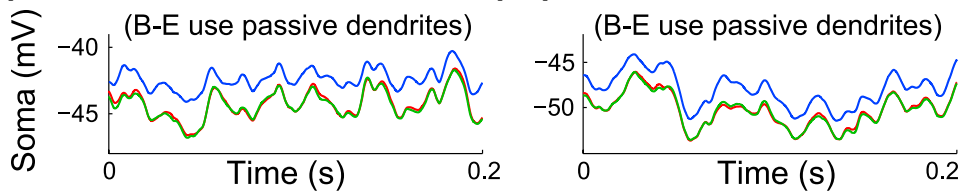

$\left(b_{3}\right)$

$\left(c_{1}\right)$

$\left(c_{2}\right)$

(c)
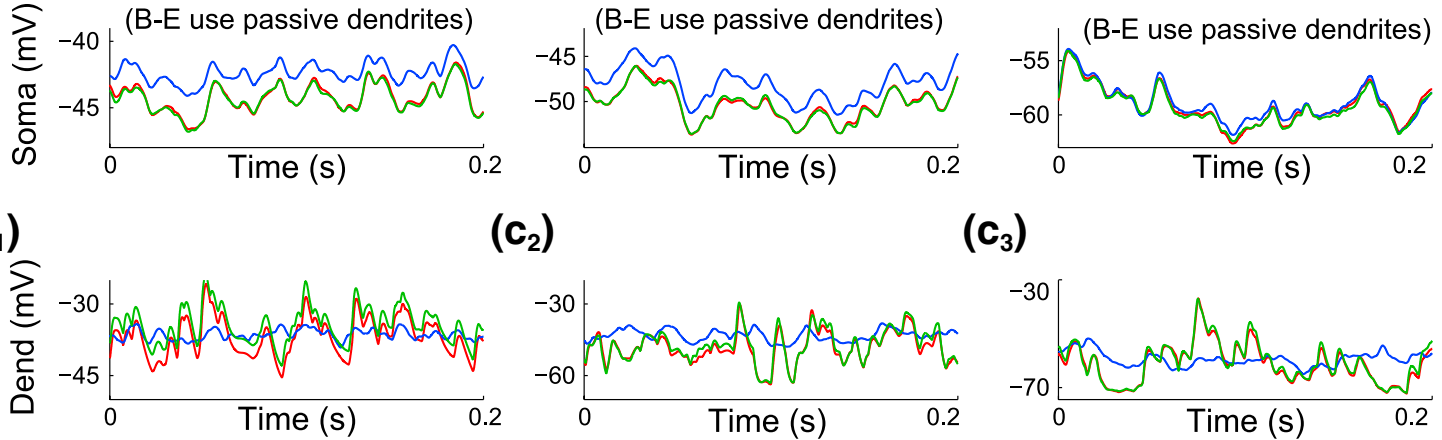

$\left(d_{1}\right)$

$\left(d_{2}\right)$

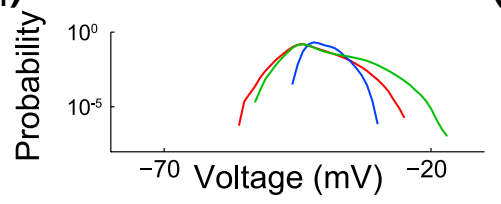

$\left(e_{1}\right)$

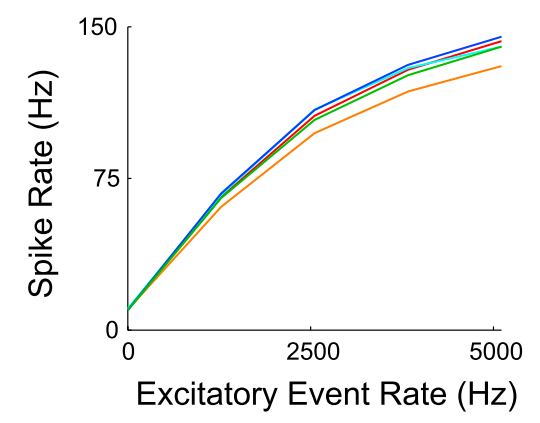

$\left(e_{2}\right)$

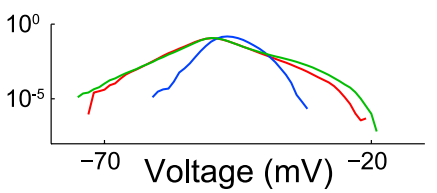

$\left(d_{3}\right)$

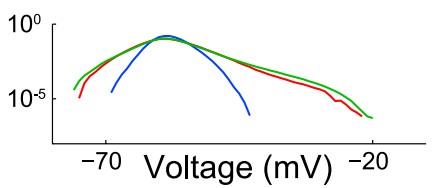

$\left(e_{3}\right)$
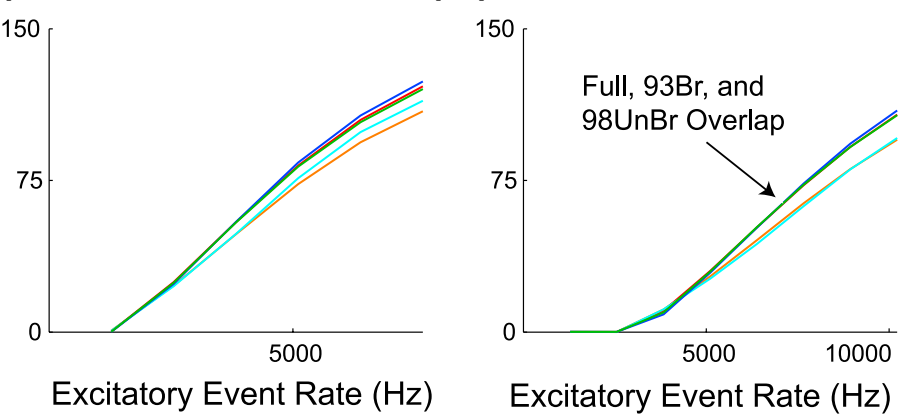

Fig. 8 The spike frequency (f) responses of the full model to different event frequencies $(\mathrm{F})$ of synaptic input (fF curves) were well matched by the divided branched reduced models but less well matched by the other reduced models. (a) With asynchronous excitation, the divided branched reduced models consistently provided the closest match to the $\mathrm{fF}$ curves of the full model (59comp model responses not shown, but similar to those of the 93 comp model). Note that the fF curves of the unbranched models were generally steeper than those of the full model, but that the mismatch depended on the precise combination of excitation and inhibition. 'No', 'Medium', and 'High' inhibition respectively refer to average rates for each inhibitory synapse of 0,10 , and $20 \mathrm{~Hz}$. Each $\mathrm{fF}$ curve was constructed from $100 \mathrm{~s}$ simulations for each data point. (b-d), somatic and axonal conductances were removed to prevent spiking. Event frequencies circle in panels $a_{1-3}$ were used for panels (b-d). (b) Short somatic voltage traces are shown in each type of model. Note that the full and branched somatic voltages were practically identical while the unbranched 98 comp model exhibited slightly different fluctuations with no or medium inhibition. Further note the correspondence of these somatic voltage fluctuations to the relative firing rates of each model. (c) Short voltage traces are shown from an example dendritic compartment at approximately the same electrotonic position and branching structure (if possible) in each type of model. The full and branched dendritic compartments experienced larger voltage fluctuations than did the compartment in the unbranched model. Note the close correspondence of the full and 93comp branched reduced model's dendritic voltage fluctuations. (d) The probability that a particular voltage was reached by any dendritic compartment at any time during synaptic input is plotted for each model. The ranges of voltages reached by the dendrites of the full and branched models were larger than the range of voltages reached by the dendrites of the unbranched model. (e) After removing all active dendritic conductances, the $\mathrm{fF}$ curves were reproduced. Note the much closer $\mathrm{fF}$ curve matches, particularly between the 98 comp unbranched reduced model and the full model 
without dendritic conductances were much more similar to each other than in the case with dendritic conductances (Fig. 8(e)).

3.8 Many of the precise spike times of the full model were preserved by the branched and unbranched reduced models

While synaptic rate coding is used for many neural computations, some circuits such as those involved with processing transient sensory inputs must use the information contained in the timing of the first spike (VanRullen et al. 2005). In network models of circuits employing precise spike timing as a coding mechanism, the exact firing times of the component neuron models therefore become important. If the spike times of a reduced branched or unbranched model differ from those of a biological neuron or full model, the degree of spike time preservation should be understood before a particular morphological reduction method is used to construct neurons for such networks. Therefore, we evaluated the preservation of precise full model spike times by branched or unbranched reduced models in the presence of identical synaptic inputs.

To study the precision of spike timing with synaptic inputs, we constructed a set of asynchronous distributed inputs where each of the 511 excitatory synapses was randomly activated with a mean rate of $5 \mathrm{~Hz}$, and each of 511 inhibitory synapses with a mean rate of $10 \mathrm{~Hz}$. After carefully examining the spike time preservation of each reduced model at each point on the $\mathrm{fF}$ curves shown in Fig. 8(a), we found that this combination of excitation and inhibition displayed a degree of spike time preservation that was representative of the range of synaptic backgrounds that we studied (e.g. Fig 8(a)). Therefore, we present the specifics of spike time preservation by the reduced models with this one synaptic background to avoid introducing redundant detail. Because input synchronization is generally thought to be an important aspect in neural coding and occurs naturally for example due to oscillatory activity (Ward 2003), we also constructed a synaptic stimulus in which clusters of synapses were activated synchronously (see Methods). Spike time preservation was defined as the percentage of the full model's spikes that were generated by the reduced model within $\pm 5 \mathrm{~ms}$ of the full model's spike time, a method we previously employed in dynamic clamp studies to examine spiking precision (Gauck and Jaeger 2000). In our previous dynamic clamp study, we showed that about $60 \%$ of precise spike times were preserved within $\pm 5 \mathrm{~ms}$ when repeatedly applying the same conductance activation pattern to neurons in slice. We consider this degree of preservation to be the limit of spike time accuracy due to intrinsic neural noise and thus any reduced models matching this level of precision present sufficient replication of full model activity.
We found that preservation of \pm 5 ms spike timing did not vary widely between the branched and unbranched reduced models (Fig. $\left.9\left(a_{1-3}\right)\right)$. Preservation was generally about $50 \%$ with asynchronous excitation (Fig. $9\left(a_{1-3}\right)$ ), and from $50 \%$ to $75 \%$ with synchronous excitation (Fig. $\left.9\left(b_{1-3}\right)\right)$, for branched or unbranched reduced models. Coincident excitatory inputs increased spike time preservation because powerful events were more likely to overcome small differences in activity between the models to drive spikes at a precise time. With spike time preservation, as with the other functional measures analyzed in this study, the dendritic gNa level was an important determinant of how well the reduced models matched the full model. Preservation of spike timing was greatly diminished by high dendritic gNa whether inputs were asynchronous or synchronous. With $10 \mathrm{~Hz}$ inhibition and $5 \mathrm{~Hz}$ excitation, spike time preservation for the high-dendritic-gNa models was often less than $20 \%$ due to differences in dendritic spike propagation and initiation with the full model (data not shown). Conversely, removing dendritic $\mathrm{gNa}$ altogether caused spike time preservation to improve by $5 \%$ relative to the case with medium dendritic $\mathrm{gNa}$ for the $93 \mathrm{comp}$ model, suggesting that dendritic sodium was amplifying coincident EPSPs differently in the different models (data not shown). Removing the remaining dendritic conductances did not further improve spike time preservation for the $93 \mathrm{comp}$ branched model, most likely because close somatic voltage fluctuation matches were observed even with all dendritic conductances present (e.g. Fig. 8(b)).

To understand why the 93comp model did not preserve $100 \%$ of precise full model spike times despite the lack of noise, identical synaptic activation patterns, and direct mapping of synaptic positions, we compared the somatic voltage responses of both models under two conditions. In, the first condition, somatic and axonal conductances were removed from both models, leaving just dendritic conductances ("nonspiking" in Fig. 9(c)). We observed a close match between the somatic responses of the full and 93comp models in the nonspiking case (Fig. 9(c), dashed traces). In the second condition, both models possessed the full complement of somatic, axonal, and dendritic conductances ("spiking" in Fig. 9(c)). In the spiking models, one model occasionally fired a spike while the other model did not quite reach threshold (e.g. Fig. 9(c), black arrow). If a spike-triggering synaptic event occurred for both models immediately after this event, then the model that just fired a spike did not fire again, while the other one did (e.g. Fig. 9(c), blue arrow). This kind of sequence tended to cause spikes in the subsequent few hundred milliseconds to be misaligned between the two models and accounted for most of the unpreserved spike times. Indeed, due to the fact that nearthreshold events can lead to spike initiation in one condition but fail to cause spike initiation in a closely parallel 


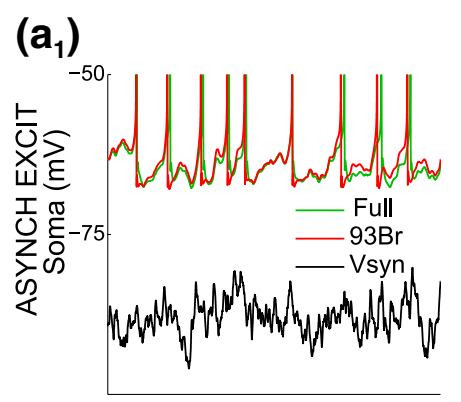

$\left(a_{2}\right)$

$\left(b_{1}\right)$

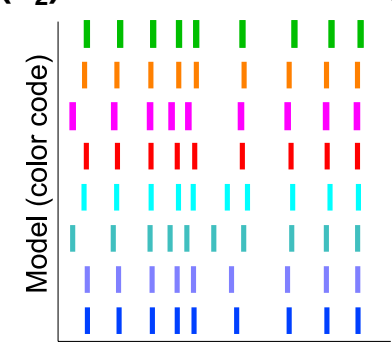

$\left(a_{3}\right)$

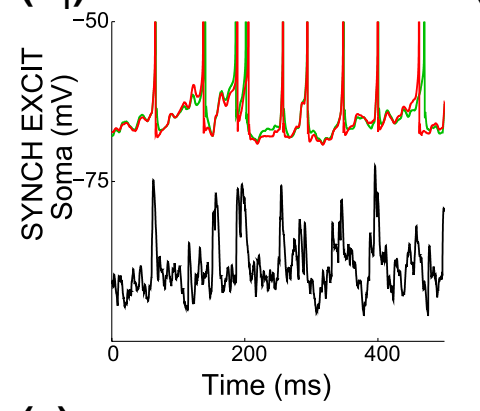

$\left(b_{2}\right)$

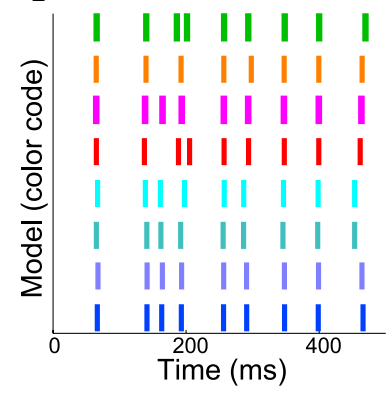

\section{$\left(b_{3}\right)$}
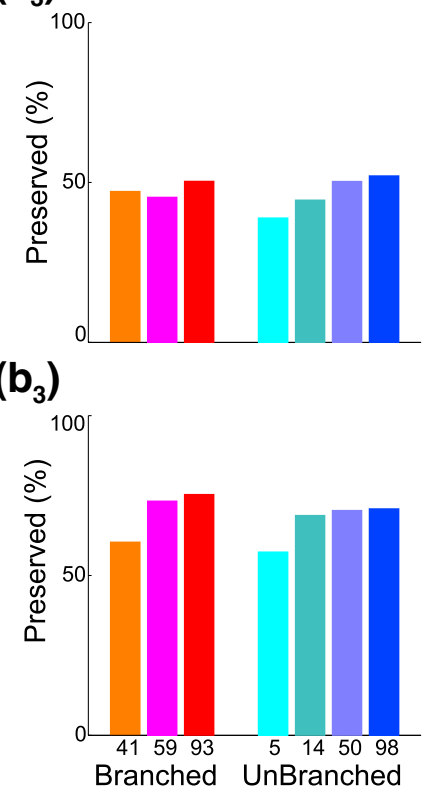

(c)

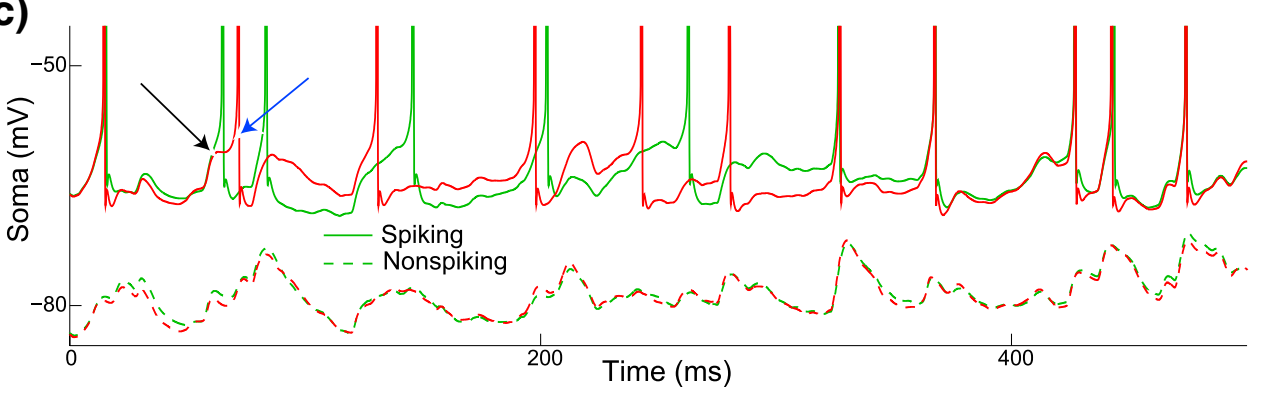

Fig. 9 Precise full model spike times were often preserved by the reduced models in the presence of synaptic input. (a) Spike time preservation during asynchronous synaptic bombardment. $a_{1}$, Somatic voltage traces for the full model and 93comp branched reduced model are shown along with the time-varying combined synaptic reversal potential (Vsyn). As we have done previously (Gauck and Jaeger 2000), Vsyn was calculated by multiplying the reversal potential of each synaptic conductance type (AMPA, NMDA, and GABA) by the momentary whole cell synaptic conductance for that type and then dividing by the total synaptic conductance for all three types. $\mathrm{V}_{\text {syn }}$ was shifted down by $20 \mathrm{mV}$ relative to voltage traces for easier visualization. $a_{2}$, Raster plot showing the spike times for each model during the simulation segment in $a_{1} . a_{3}$, The percent of preserved spikes was generally about $50 \%$ for all the reduced models. (b) Same as (a), but with synchronous excitation. Note the improved preservation percentages for each reduced model. Further note that none of the reduced models preserved more than about $75 \%$ of full model spike

condition, a fully precise control of spike timing by synaptic input may be impossible.

Overall, in the absence of dendritic spiking, the degree of precise full model spike time preservation by the reduced models was similar to the spike time preservation that we have observed between multiple applications of identical conductance activation patterns to slice neurons (Gauck and Jaeger 2000). times. (c) Somatic voltage traces are shown in the full and 93comp models during a short period when precise full model spike times were not preserved. The dashed traces represent the somatic voltages of the models without somatic or axonal conductances (nonspiking, shifted down by $20 \mathrm{mV}$ to aid visualization), while the solid traces are from the active models. Note the extremely close match of the nonspiking somatic voltage fluctuations, the point when the full model spikes but the 93comp model does not (black arrow), and the 93comp spike which follows soon after (blue arrow). Synaptic input distributions were identical to those used for Fig. 8. Medium excitation $(5 \mathrm{~Hz})$ and medium inhibition $(10 \mathrm{~Hz})$ were used for all panels of this figure. Preservation of full model spike times was calculated as the percentage of the full model's spikes that were generated by the reduced model within $\pm 5 \mathrm{~ms}$ of the full model's spike time. The colors used in the raster plots in this figure were the same as those used for the rest of the figures

3.9 Conductance parameter sets yielding disparate output can be used in the full and reduced models to produce similar output

Researchers using full models commonly face the problem of hand-tuning conductance densities to match target output: this is always a laborious task and dissatisfying in that only a single - possibly arbitrary — solution is found. 
Therefore, there has been great interest in finding algorithms to optimize the conductance densities of a model automatically (Huys et al. 2006; Vanier and Bower 1999) and to determine the parameter space of similar solutions with database approaches (Gunay et al. 2008; Prinz et al. 2003; Prinz et al. 2004). However, even with such algorithms it is still demanding to find a suitable parameter set for a full model because of the long simulation times required to run thousands of parameter combinations in such models. This time requirement can be drastically decreased by applying the search algorithm to a reduced model, as long as the
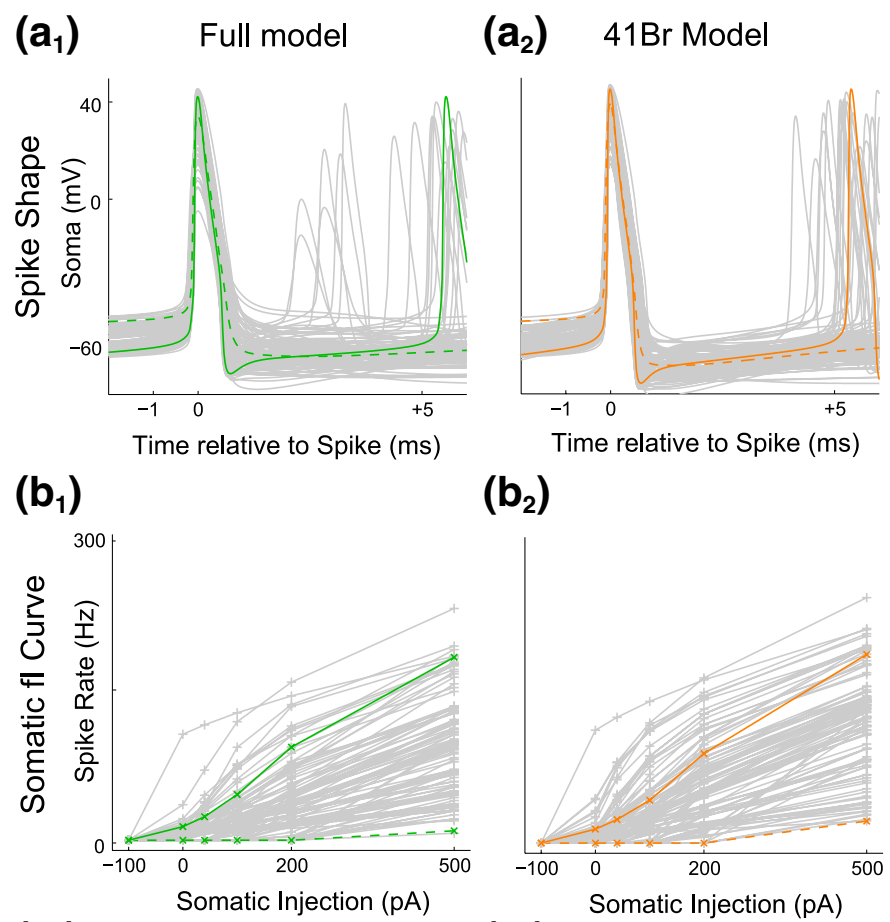

$\left(b_{2}\right)$
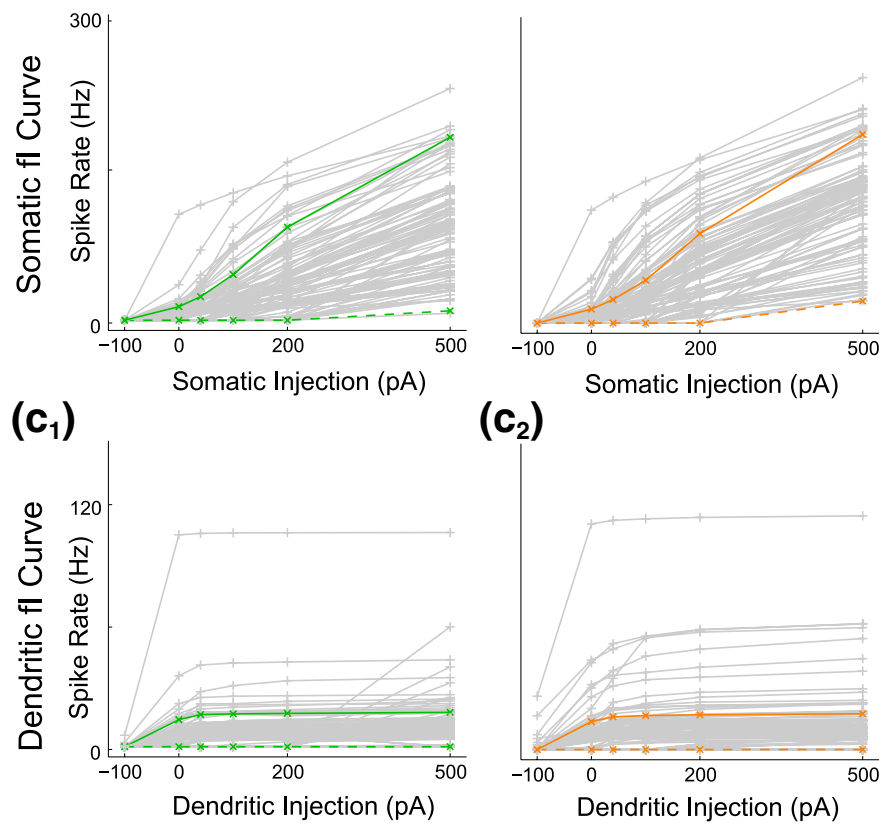

$\left(c_{2}\right)$

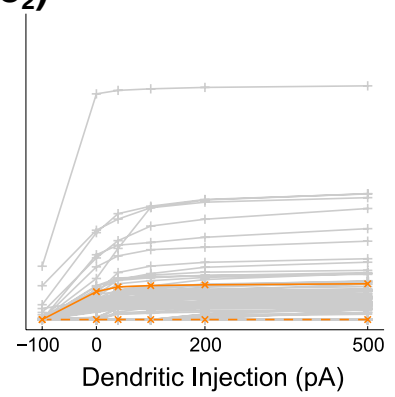

Fig. 10 Different model activity patterns generated by many random conductance density sets were matched between the full and reduced models. One hundred conductance density parameter sets were randomly generated by varying each conductance density between $25 \%$ and $400 \%$ of its original value. Two of these parameter sets that produced very different model outputs were selected to illustrate the responses of the different models with identical conductance densities (colored solid or dashed lines). The remaining 98 random parameter sets are displayed as solid light gray lines. Model spike shapes were compared during $500 \mathrm{pA}$ somatic DC injection because this level of current injection caused every morphology to spike with each random resulting conductance densities can be used in the full model to produce similar output.

To examine which reduction strategy could be used to efficiently search the parameter space of the full model, 100 random parameter sets were generated by varying each conductance density independently between $25 \%$ and $400 \%$ of its default value. These parameter sets produced widely varying spike shapes as well as somatic and dendritic fI curves (Fig. 10). Of these parameter sets, two that yielded different somatic and dendritic fI curves and spike shapes were chosen to illustrate a comparison of the output of the
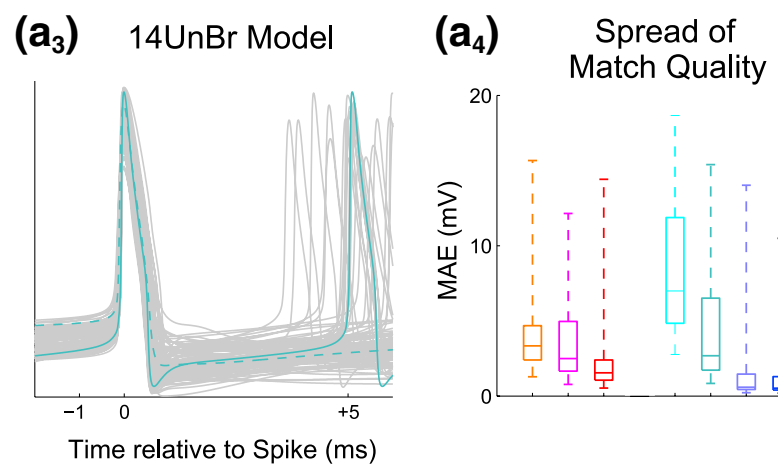

$\left(b_{3}\right)$
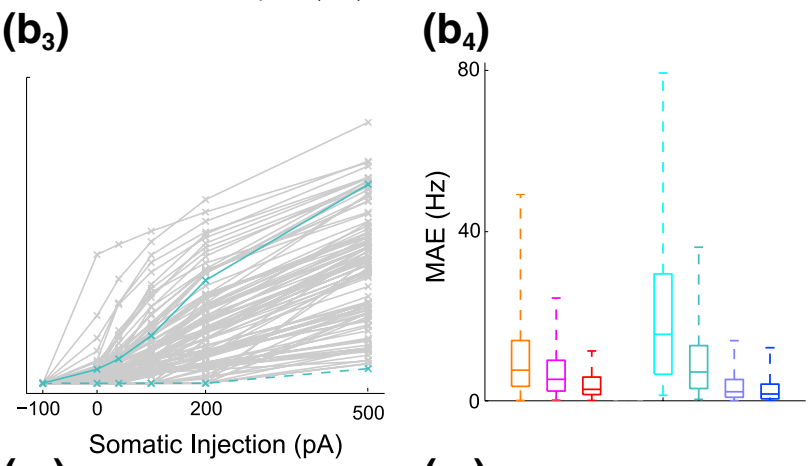

$\left(\mathbf{c}_{3}\right)$

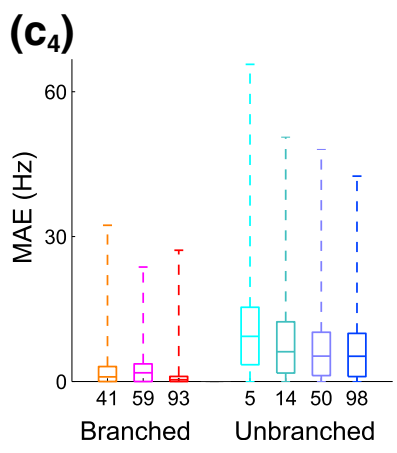

parameter set tested. $\left(a_{1}, b_{1}, c_{1}\right)$, Each random parameter set was inserted into the full model. For each parameter set, the somatic spike shape $\left(a_{1}\right)$, somatic fI curve $\left(b_{1}\right)$ and dendritic fI curve $\left(c_{1}\right)$ are displayed. Note the wide range of spike shapes and fI curve gains. $\left(a_{2}\right.$, $\left.b_{2}, c_{2}\right)$, Same as the first column but for the 41 comp branched morphology. $\left(a_{3}, b_{3}, c_{3}\right)$, Same as the first two columns but for the 14comp unbranched morphology. $\left(a_{4}, b_{4}, c_{4}\right)$, Box plots of the distribution of MAEs calculated for the 100 random parameter sets are shown for each reduced model. Note that dendritic fI curve MAEs were smaller for the branched reduced models than for the unbranched models 
full and reduced models (Fig. 10, 'RandSet1' (colored solid lines) and 'RandSet2' (colored dashed lines); for conductance densities see Supplemental Table S3, Online Resource 1). This comparison showed that the distinct differences in the model output between the two parameter sets were generally preserved in both branched and unbranched models (Fig. 10). For instance, the somatic fI curves and spike shapes were closely preserved when these two parameter sets were used in the full, 41comp branched and 14comp unbranched models (Fig. 10(a,b)). Note that even the $5 \mathrm{~ms}$ ISI doublet firing of the full model with RandSet1 (colored solid lines) was preserved by both reduced models (Fig. 10 (a)). In contrast, the dendritic fI curves were closely preserved by the 41 comp branched model but not by the 14comp unbranched model, which generally fired faster with the same current injection input (Fig. 10(c)).

To quantify the ability of the reduced and full models to produce similar output with the same densities across all 100 parameter sets, we calculated the MAE between the full and reduced model output for each measure (6 rate points for each fI curve and 801 voltage points for each spike shape). This yielded a set of 100 MAEs for each measure in each reduced model. We observed that increasing the number of compartments provided significantly smaller somatic spike shape and somatic fI MAEs $(p<0.05$ with Bonferroni correction using the Mann-Whitney U test) for both branched and unbranched reduced models in most cases (Fig. $10\left(a_{4}, b_{4}\right)$ ) see Supplemental Table S4 for all statistical comparisons, Online Resource 1). In contrast, increasing the number of compartments while keeping the reduced model type fixed significantly decreased the median dendritic fI MAE in only one instance (from 59comp to $93 \mathrm{comp}$ branched reduced, Supplemental Table S4, Online Resource 1). Instead of a compartment number relationship, we observed that preserved branching structure allowed much smaller MAEs for the branched reduced models than for the unbranched reduced models regarding the dendritic fI curve. For example, the median dendritic fI curve MAE exhibited by the 93comp branched reduced model was significantly ( $p=$ 1.9e-11, Mann-Whitney U test) smaller by $4.9 \mathrm{~Hz}$ than that exhibited by the 98 comp unbranched reduced model, despite having fewer compartments (Supplemental Table S4, Online Resource 1). The differing ability of the branched and unbranched reduced models to reproduce full model dendritic responses with identical conductance densities was very similar to the differing ability of the two types of reduced models to match the dendritic responses of the full model with the tuned parameter set (Fig. 6). The similar deviations between models for these 100 randomly generated parameter sets support the general validity of our analysis tracing these differences back to axial current and dendritic $\mathrm{R}_{\mathrm{IN}}$ mismatches.

Overall, we found that identical conductance densities allowed our unbranched reduced models to produce somatic input output dynamics similar to those of the full model, while the branched reduced models additionally reproduced the dendritic input driven somatic output dynamics of the full model.

\section{Discussion}

We developed a principled strategy to construct branched or unbranched reduced dendritic neuron models with few compartments and multiple active conductances that allowed us to keep the same channel densities as used in a full morphological model. Using these models, we demonstrate that full model responses to somatic stimuli can be closely matched by both branched and unbranched reduced models. In contrast, because of unavoidable differences in the local dendritic input resistance of unbranched reduced model dendrites, responses to dendritic input are less closely matched by this type of model if dendrites contain voltage-gated currents, even if the remaining major dendrites are subdivided into many compartments. Some full model properties could not be fully matched even by branched reduced models, in particular when it came to matching either bAP amplitudes with medium dendritic gNa or spiking responses to synaptic input with high dendritic $\mathrm{gNa}$. We found that particular aspects of the passive structure of reduced models explained particular unavoidable mismatches with full model activity. Specifically, the lumping of axial resistances in models with few compartments led to mismatches in spike shapes and fI curves, while local dendritic $R_{I N}$ and $Z_{\mathrm{IN}}$ differences led to mismatches in responses to dendritic stimuli and bAP amplitudes.

Despite these remaining mismatches, we show that many aspects of full model activity, including rate and time coding with dendritic inputs, can be well replicated by principled model reduction strategies and that different sets of voltage gated conductances result in similar activity patterns for each type of model.

\subsection{Principles of morphological reduction}

In passive models, many precise input responses can be maintained if the dendritic tree of a full model meets two criteria: 1) all branches follow the $3 / 2$ power branching rule and 2) there is equal electrotonic length from the soma to each dendritic tip (Rall 1959, 1964). Like most biological dendritic trees, the dendritic tree of the full GP model did not meet either of these criteria. The passive properties of this type of full model cannot be entirely preserved by morphologically reduced models with altered electrotonic structure (Holmes and Rall 1992). Preservation of dynamics becomes even more complicated with the addition of voltage and time dependent conductances to the membrane (Rall et al. 1992). Despite the need for a clear understanding of 
active reduced model capabilities due to their widespread adoption by modelers, it is not yet clear which active properties can and cannot be maintained following morphological reduction. Because an analysis of active model reductions is not amenable to purely mathematical techniques, compartmental modeling presents the best tool to explore relationships between the dynamics of full and reduced morphologies.

To understand the extent to which active full model dynamics can be preserved by morphologically reduced models, we chose to examine the consequences of two particular morphological reduction methods to create simplified dendritic neuron models, one that preserved detailed dendritic branching structure and one that did not. Different strategies have been employed among previously used dendritic morphological reduction methods, usually without a detailed examination of the consequences. Some studies use reduced models that maintain only a qualitative relationship between full and reduced morphologies without preserving any particular morphological features (Davison et al. 2000; Traub et al. 1991; Wilson and Bower 1992). Another study examined the reduction of a full model with somatic but not dendritic conductances carefully, and described a method that conserves the full model Ra at the expense of TSA (Bush and Sejnowski 1993). More recent studies have employed reduction methods that preserve the TSA and $\mathrm{R}_{\mathrm{IN}}$ of the full model while ignoring its $\mathrm{Ra}$ (Destexhe 2001; Tobin et al. 2006). We maintained this strategy as our reduced models preserved full model TSA and approximated somatic $\mathrm{R}_{\mathrm{IN}}$ when compartments of the most reduced models were sequentially subdivided. In addition, we preserved the electrotonic length of major dendritic branches (our unbranched models) or of every dendritic branchlet (our branched models). Our method, as with the methods of Tobin and Destexhe, can be used to construct reduced models both from previously existing full models, but also to construct reduced models directly from reconstructed morphologies and electrophysiological data. Furthermore, our method, like that of Tobin et al, allows channel densities to be used in both full and reduced models to produce similar electrophysiological activity with somatic stimulation provided that there are a sufficient number of reduced model compartments. This capability allows our reduced models with $>10$ compartments to be used as fast search engines of full model parameter space.

\subsection{The suitability of different levels of model reduction} for different applications

\subsubsection{If complex dendritic processing is not required, then unbranched reduced models suffice}

Computational efficiency is an important consideration when choosing which neuron models to incorporate into large networks, and in this respect unbranched reduced models are preferable to branched reduced models because far fewer compartments are needed to yield satisfactory matches to somatic input-output functions. For example, the unbranched reduced models presented here, particularly the 14 comp model, could be incorporated into existing GP network models which currently use single compartment or simpler models (Rubchinsky et al. 2003; Terman et al. 2002). These models replicated the rate coding function for somatic current injection well without altering the channel densities of the full morphological model. Thus, they would allow the study of how individual voltage-gated conductances may contribute to important features of somatic processing. Of course, if mathematical tractability of analyzing network activity patterns is desired, then even more simplified neuron models such as exponential integrate and fire neurons or low dimensional dynamical system models are preferable (Herz et al. 2006; Izhikevich 2004).

\subsubsection{Network models in need of accurate synaptic coding with active dendritic conductances can employ branched reduced models}

We showed that branched reduced models can closely match the spiking responses of the full model to dendritic current injection or synaptic input, while the unbranched reduced models provided less precise matches. However, the matches even of branched reduced models became significantly worse if a high level of dendritic gNa allowed dendritic spike initiation. Overall, we found no reduced model that could accurately reproduce the pattern of dendritic spike initiation shown by the full model, since this response is highly sensitive to the amplitude of local dendritic voltage fluctuations, which are not generally matched by reduced models due to unavoidable deviations in local input resistances. In contrast, bAPs could be closely matched in branched reduced models, although in a particular regime of intermediate dendritic $\mathrm{gNa}$ densities some branches showed increased amplification of bAPs only in the full model. The interaction of bAPs is often considered important for spike time dependent plasticity rules (Gulledge et al. 2005; Letzkus et al. 2006), which could therefore generally be employed in network models using branched reduced models, while unbranched models would be less suitable (though not completely inaccurate).

\subsubsection{Applications that involve locally non-linear dendritic processing should only use full models}

Our analysis of the dendritic properties of reduced and full models revealed that dendritic $R_{I N} S$, and especially $Z_{I N} S$ for brief or fast-changing signals, cannot be matched for all regions of the dendrite in any model reduction. The local 
dendritic $Z_{\mathrm{IN}} \mathrm{S}$ determine the local voltage response to synaptic input and therefore directly influence the amount of activation of voltage-gated conductances. This is particularly apparent in models that allow dendritic spike initiation due to a high dendritic $\mathrm{gNa}$, but also becomes important for fast transient events like bAPs.

\subsubsection{Using reduced models to search the channel density parameter space}

Several studies have shown that it is of significant interest to explore the parameter space of full morphological models through evolutionary algorithms (Achard and De Schutter 2006) or database methods (Gunay et al. 2008; Prinz et al. 2003). A large number of simulation runs are also required to determine the sensitivity of a model to channel density variations (Weaver and Wearne 2008). These methods are computationally expensive to carry out in full morphological models. Because our model reduction method was designed so that full model channel densities can be used to produce similar dynamics in the reduced model, it can increase the efficiency of parameter space exploration by $10-100$ fold.

\section{Conclusion}

Reduced models are widely used, particularly in network simulations due to their computational efficiency. Careful validation of dendritic activity in reduced models is likely important to achieve biologically realistic processing in large network simulations. Our findings indicate that carefully constructed reduced models with 10-50 times fewer compartments than a full morphological model can accurately reproduce somatic spontaneous and most synaptically driven activities, while extremely simplified 1-5 compartment models cannot. While some deviations from full morphological models were found for all reduced models, these were generally no larger than the biological variability of the same features in the same cell type. The detailed dendritic branch structure made important contributions to processing only in the presence of strong dendritic non-linearities, such as local $\mathrm{Na}^{+}$spike initiation, and should be considered in models employing such mechanisms.

Acknowledgements This project was supported by National Institute of Neurological Disorders and Stroke (NINDS) grant R01-NS039852 to DJ and National Science Foundation (NSF) DGE-0333411 IGERT fellowship to EBH.

Conflict of Interest Notification To the best of our knowledge, no conflicts of interests exist related to the work we present here.
Open Access This article is distributed under the terms of the Creative Commons Attribution Noncommercial License which permits any noncommercial use, distribution, and reproduction in any medium, provided the original author(s) and source are credited.

\section{References}

Achard, P., \& De Schutter, E. (2006). Complex parameter landscape for a complex neuron model. PLoS Computational Biology, 2, 794-804.

Bean, B. P. (2007). The action potential in mammalian central neurons. Nature Reviews. Neuroscience, 8, 451-465.

Burke, R. E. (2000). Comparison of alternative designs for reducing complex neurons to equivalent cables. Journal of Computational Neuroscience, 9, 31-47.

Bush, P. C., \& Sejnowski, T. J. (1993). Reduced compartmentalmodels of neocortical pyramidal cells. Journal of Neuroscience Methods, 46, 159-166.

Caporale, N., \& Dan, Y. (2008). Spike timing-dependent plasticity: a Hebbian learning rule. Annual Review of Neuroscience, 31, 25-46.

Davison, A. P., Feng, J. F., \& Brown, D. (2000). A reduced compartmental model of the mitral cell for use in network models of the olfactory bulb. Brain Research Bulletin, 51, 393-399.

Destexhe, A. (2001). Simplified models of neocortical pyramidal cells preserving somatodendritic voltage attenuation. Neurocomputing, $38,167-173$.

Destexhe, A., Neubig, M., Ulrich, D., \& Huguenard, J. (1998). Dendritic low-threshold calcium currents in thalamic relay cells. The Journal of Neuroscience, 18, 3574-3588.

Destexhe, A., Rudolph, M., \& Pare, D. (2003). The high-conductance state of neocortical neurons in vivo. Nature Reviews. Neuroscience, 4, 739-751.

Gauck, V., \& Jaeger, D. (2000). The control of rate and timing of spikes in the deep cerebellar nuclei by inhibition. The Journal of Neuroscience, 20, 3006-3016.

Golding, N. L., \& Spruston, N. (1998). Dendritic sodium spikes are variable triggers of axonal action potentials in hippocampal CA1 pyramidal neurons. Neuron, 21, 1189-1200.

Gulledge, A. T., Kampa, B. M., \& Stuart, G. J. (2005). Synaptic integration in dendritic trees. Journal of Neurobiology, 64, 75-90.

Gunay, C., Edgerton, J. R., \& Jaeger, D. (2008). Channel density distributions explain spiking variability in the globus pallidus: a combined physiology and computer simulation database approach. The Journal of Neuroscience, 28, 7476-7491.

Hanson, J. E., Smith, Y., \& Jaeger, D. (2004). Sodium channels and dendritic spike initiation at excitatory synapses in globus pallidus neurons. The Journal of Neuroscience, 24, 329-340.

Herz, A. V. M., Gollisch, T., Machens, C. K., \& Jaeger, D. (2006). Modeling single-neuron dynamics and computations: a balance of detail and abstraction. Science, 314, 80-85.

Holmes, W. R., \& Rall, W. (1992). Estimating the electrotonic structure of neurons with compartmental-models. Journal of Neurophysiology, 68, 1438-1452.

Holmes, W. R., \& Woody, C. D. (1989). Effects of uniform and non-uniform synaptic activation-distributions on the cable properties of modeled cortical pyramidal neurons. Brain Research, 505, 12-22.

Huys, Q. J. M., Ahrens, M. B., \& Paninski, L. (2006). Efficient estimation of detailed single-neuron models. Journal of Neurophysiology, 96, 872-890.

Izhikevich, E. M. (2004). Which model to use for cortical spiking neurons? IEEE Transactions on Neural Networks, 15, 1063-1070. 
Johnston, D., \& Wu, S. M. S. (1995). Foundations of cellular neurophysiology. Cambridge: MIT.

Kuznetsov, A. S., Kopell, N. J., \& Wilson, C. J. (2006). Transient highfrequency firing in a coupled-oscillator model of the mesencephalic dopaminergic neuron. Journal of Neurophysiology, 95, 932-947.

Letzkus, J. J., Kampa, B. M., \& Stuart, G. J. (2006). Learning rules for spike timing-dependent plasticity depend on dendritic synapse location. The Journal of Neuroscience, 26, 10420-10429.

London, M., \& Hausser, M. (2005). Dendritic computation. Annual Review of Neuroscience, 28, 503-532.

Losonczy, A., Makara, J. K., \& Magee, J. C. (2008). Compartmentalized dendritic plasticity and input feature storage in neurons. Nature, 452, 436-U433.

McCormick, D. A., Shu, Y. S., \& Yu, Y. G. (2007). Hodgkin and Huxley model - still standing? Nature, 445, E1-E2.

Naundorf, B., Wolf, F., \& Volgushev, M. (2006). Unique features of action potential initiation in cortical neurons. Nature, 440, 1060-1063.

Prinz, A. A., Billimoria, C. P., \& Marder, E. (2003). Alternative to hand-tuning conductance-based models: construction and analysis of databases of model neurons. Journal of Neurophysiology, 90, 3998-4015.

Prinz, A. A., Bucher, D., \& Marder, E. (2004). Similar network activity from disparate circuit parameters. Nature Neuroscience, 7, 1345-1352.

Rall, W. (1959). Branching dendritic trees and motoneuron membrane resistivity. Experimental Neurology, 1, 491-527.

Rall, W. (1964). Theoretical significance of dendritic trees for neuronal input-output relations. In R. F. Reiss (Ed.), Neural theory and modeling (pp. 73-97). Stanford: Stanford University Press.

Rall, W., Burke, R. E., Holmes, W. R., Jack, J. J. B., Redman, S. J., \& Segev, I. (1992). Matching dendritic neuron models to experimental-data. Physiological Reviews, 72, S159-S186.

Rubchinsky, L. L., Kopell, N., \& Sigvardt, K. A. (2003). Modeling facilitation and inhibition of competing motor programs in basal ganglia subthalamic nucleus-pallidal circuits. Proceedings of the National Academy of Sciences of the United States of America, $100,14427-14432$.

Schaefer, A. T., Larkum, M. E., Sakmann, B., \& Roth, A. (2003). Coincidence detection in pyramidal neurons is tuned by their dendritic branching pattern. Journal of Neurophysiology, 89, 3143-3154.

Terman, D., Rubin, J. E., Yew, A. C., \& Wilson, C. J. (2002). Activity patterns in a model for the subthalamopallidal network of the basal ganglia. The Journal of Neuroscience, 22, 2963-2976.

Tobin, A. E., Van Hooser, S. D., \& Calabrese, R. L. (2006). Creation and reduction of a morphologically detailed model of a leech heart interneuron. Journal of Neurophysiology, 96, 2107-2120.

Traub, R. D., Kopell, N., Bibbig, A., Buhl, E. H., LeBeau, F. E. N., \& Whittington, M. A. (2001). Gap junctions between interneuron dendrites can enhance synchrony of gamma oscillations in distributed networks. The Journal of Neuroscience, 21, 9478-9486.

Traub, R. D., Wong, R. K. S., Miles, R., \& Michelson, H. (1991). A model of a CA3 hippocampal pyramidal neuron incorporating voltageclamp data on intrinsic conductances. Journal of Neurophysiology, 66, 635-650.

Vanier, M. C., \& Bower, J. M. (1999). A comparative survey of automated parameter-search methods for compartmental neural models. Journal of Computational Neuroscience, 7, 149-171.

VanRullen, R., Guyonneau, R., \& Thorpe, S. J. (2005). Spike times make sense. Trends in Neurosciences, 28, 1-4.

Ward, L. M. (2003). Synchronous neural oscillations and cognitive processes. Trends in Cognitive Sciences, 7, 553-559.

Weaver, C. M., \& Wearne, S. L. (2008). Neuronal firing sensitivity to morphologic and active membrane parameters. PLoS Computational Biology, 4, e11.

Wilson, M., \& Bower, J. M. (1992). Cortical oscillations and temporal interactions in a computer-simulation of piriform cortex. Journal of Neurophysiology, 67, 981-995. 